Canad. J. Math. Vol. 71 (1), 2019 pp. 153-182

http://dx.doi.org/10.4153/CJM-2018-013-8

(c) Canadian Mathematical Society 2018

\title{
Weighted Distribution of Low-lying Zeros of GL(2) L-functions
}

\author{
Andrew Knightly and Caroline Reno
}

\begin{abstract}
We show that if the zeros of an automorphic $L$-function are weighted by the central value of the $L$-function or a quadratic imaginary base change, then for certain families of holomorphic GL(2) newforms, it has the effect of changing the distribution type of low-lying zeros from orthogonal to symplectic, for test functions whose Fourier transforms have sufficiently restricted support. However, if the $L$-value is twisted by a nontrivial quadratic character, the distribution type remains orthogonal. The proofs involve two vertical equidistribution results for Hecke eigenvalues weighted by central twisted $L$-values. One of these is due to Feigon and Whitehouse, and the other is new and involves an asymmetric probability measure that has not appeared in previous equidistribution results for $\mathrm{GL}(2)$.
\end{abstract}

\section{Introduction}

According to the density conjecture of Katz and Sarnak, for any suitable family of $L$-functions, the zeros lying close to the real axis are equidistributed according to one of a handful of possible symmetry types coming from compact classical groups ( [KS1, KS2]). More precisely, given an $L$-function $L(s, f)$, denote its nontrivial zeros by $\rho_{f}=$ $\frac{1}{2}+i \gamma_{f}$, and define the 1-level density

$$
\mathcal{D}(f, \phi)=\sum_{\rho_{f}} \phi\left(\frac{\gamma_{f} \log Q_{f}}{2 \pi}\right),
$$

where $Q_{f}$ is the analytic conductor of $f$, and $\phi$ is a test function. The conjecture predicts that for any suitable family $\mathcal{F}=\cup \mathcal{F}_{n}$ of automorphic forms, with each $\mathcal{F}_{n}$ finite, there exists a family $G$ of classical compact groups (being one of $\mathrm{O}, \mathrm{SO}$ (even), SO(odd), Sp, or U) such that for any even Schwartz function $\phi$ with compactly supported Fourier transform $\widehat{\phi}$,

$$
\lim _{n \rightarrow \infty} \frac{\sum_{f \in \mathcal{F}_{n}} \mathcal{D}(f, \phi)}{\left|\mathcal{F}_{n}\right|}=\int_{-\infty}^{\infty} \phi(x) W_{G}(x) d x .
$$

Here, $W_{G}(x)$ is the limiting distribution of the 1-level density attached to the eigenvalues of random matrices in $G$ as the rank tends to $\infty$. Of particular relevance to us here are

$$
W_{\mathrm{O}}(x)=1+\frac{1}{2} \delta_{0}(x) \text { and } W_{\mathrm{Sp}}(x)=1-\frac{\sin (2 \pi x)}{2 \pi x},
$$

Received by the editors May 10, 2017; revised February 23, 2018.

Published electronically December 18, 2018.

This work was partially supported by grant \#317659 from the Simons Foundation to author A. K. Section 3 is based in part on the University of Maine MA thesis of author C. R.

AMS subject classification: 11M41, 11F11, 11M26.

Keywords: low-lying zero, L-function. 
where $\delta_{0}$ is the Dirac distribution at 0 . As a distribution, $W_{\mathrm{Sp}}(x)$ coincides with $1-$ $\frac{1}{2} \delta_{0}(x)$ when, as will always be the case for us here, $\widehat{\phi}$ is supported in $(-1,1)$. This is a consequence of the Plancherel formula ( [ILS, (1.34)]).

Averages involving automorphic forms are naturally studied using the trace formula. Many variants of the trace formula involve weighting factors, such as the harmonic weight $\left|a_{f}(1)\right|^{2} /\|f\|^{2}$ that arises in the Petersson formula. In some cases, including that of GL(2) newforms, the presence of this weight is innocuous in the sense that it does not affect the distribution of low-lying zeros [Mi]. However, in the case of zeros of GSp(4) spinor $L$-functions, Kowalski, Saha, and Tsimmerman found that the analogous harmonic weight leads to a symplectic distribution, despite a heuristic suggesting that the unweighted distribution is orthogonal [KST]. They gave a striking interpretation of this as evidence for Böcherer's conjecture, according to which the Fourier coefficient arising in the weight contains arithmetic information in the form of central $L$-values.

The question thus arises: in the simplest case of holomorphic GL(2) cusp forms, if we weight the low-lying zeros by central $L$-values, does it likewise change the distribution from orthogonal to symplectic? The answer depends on the type of $L$-function used in the weight, as we illustrate below using several families with suitably restricted test functions. We do not use the Petersson formula, but rather the relative trace formulas developed in [FW, JK], in which central $L$-values appear directly.

In Theorem 1.1, we consider the effect of weighting by the central $L$-value and a Fourier coefficient. We show for two different families of holomorphic newforms that the weighted distribution of low-lying zeros is symplectic when $\widehat{\phi}$ is supported in $\left(-\frac{1}{2}, \frac{1}{2}\right)$. However, if the $L$-value is twisted by a nontrivial quadratic character, the weighted distribution is orthogonal. In Theorem 1.3, we show that the zeros of $L$-functions attached to newforms of prime level $N \rightarrow \infty$, when weighted by an imaginary quadratic base change central $L$-value, have symplectic distribution for $\widehat{\phi}$ supported in $(-1,1)$. We do not assume any version of the Generalized Riemann Hypothesis, though it motivates the definition of one-level density, and its use can enable one to extend the allowable range of support of $\widehat{\phi}$ ( [BBDDM], [ILS]). Of course, it would be of interest to widen the range of support beyond $(-1,1)$, because the nature of the measure $W_{\mathrm{sp}}$ changes there.

Theorem 1.1 Let $\chi$ be a primitive real Dirichlet character of modulus $D \geq 1$. Let $r>0$ be an integer relatively prime to $D$. For a holomorphic newform $f(z)=\sum a_{f}(n) e^{2 \pi i n z}$, define the weight

$$
w_{f}=\frac{\Lambda\left(\frac{1}{2}, f \times \chi\right)\left|a_{f}(r)\right|^{2}}{\|f\|^{2}}
$$

for the completed L-function $\Lambda(s, f \times \chi)$ defined in (2.3) below. Let $\phi$ be any even Schwartz function whose Fourier transform $\widehat{\phi}(y)=\int_{-\infty}^{\infty} \phi(x) e^{-2 \pi i x y} d x$ is supported inside $\left(-\frac{1}{2}, \frac{1}{2}\right)$. Then

$$
\lim _{n \rightarrow \infty} \frac{\sum_{f \in \mathcal{F}_{n}} \mathcal{D}(f, \phi) w_{f}}{\sum_{f \in \mathcal{F}_{n}} w_{f}}= \begin{cases}\int_{-\infty}^{\infty} \phi(x) W_{\mathrm{Sp}}(x) d x, & \chi \text { trivial, } \\ \int_{-\infty}^{\infty} \phi(x) W_{\mathrm{O}}(x) d x, & \chi \text { nontrivial },\end{cases}
$$


in each of the following situations:

- $n=k$ and $\mathcal{F}_{n}=F_{k}(1)$ is the set of newforms of level 1 with the weight $k$ ranging over even numbers satisfying $\tau(\chi)^{2} \neq-i^{k} D$ for the Gauss sum $\tau(\chi)=$ $\sum_{m=1}^{D} \chi(m) e^{2 \pi i m / D}$.

- $\mathcal{F}_{n}=\mathcal{F}_{k}(N)^{\text {new }}$ (with $N+k \rightarrow \infty$ as $n \rightarrow \infty$ ) is the set of newforms of prime level $N+r D$, and even weight $k>2$ chosen so that $\tau(\chi)^{2}=-i^{k} D$, or equivalently, $\chi(-1)=-i^{k}$.

Remarks 1.2 (i) Iwaniec, Luo, and Sarnak showed that in the unweighted case, the distribution is orthogonal [ILS].

(ii) We prove Theorem 1.1 in Section 4. It is shown there that in the second case, if $k$ is fixed and $N \rightarrow \infty$, the allowable support of $\widehat{\phi}$ can be widened to $[-\alpha, \alpha]$ for any $0<\alpha<1-\frac{1}{k}$.

(iii) The weights $w_{f}$ are nonnegative by Guo's theorem, [Gu]. In Section 4 , we also show that the statement of Theorem 1.1 remains true if we instead use the weight

$$
w_{f}=\frac{\Lambda\left(\frac{1}{2}, f \times \chi\right) \overline{a_{f}(r)}}{\|f\|^{2}},
$$

which may be negative. (Hypotheses on $\mathcal{F}_{n}$ imply that $a_{f}(r)$ is real here, though elsewhere in this paper it may be complex.)

(iv) The conditions involving $\tau(\chi)$ come from the functional equation (2.4) when $N=1$. Since $\chi=\bar{\chi}$, the condition $\left(i^{k} \tau(\chi)^{2}\right) / D=-1$ forces the $L$-function to vanish at $s=\frac{1}{2}$. In the first case above (where $N=1$ ), the given condition keeps this from happening and guarantees that the sum of the weights is nonzero when $k$ is sufficiently large. In the second case, where $N$ is prime, the given condition is desirable, since it causes the weights attached to the oldforms to vanish, leaving us with an expression involving only newforms.

Theorem 1.3 Fix a quadratic discriminant $-D<0$, and let $\chi=\chi_{-D}$ be the associated primitive quadratic Dirichlet character of conductor D. Let $\mathcal{F}_{N}=\mathcal{F}_{k}(N)^{\text {new }}$ be the set of holomorphic newforms of prime level $N$ and fixed even weight $k>2$. For $f \in \mathcal{F}_{N}$, define the weight

$$
w_{f}=\frac{\Lambda\left(\frac{1}{2}, f \times \chi\right) \Lambda\left(\frac{1}{2}, f\right)}{\|f\|^{2}} .
$$

Then for any even Schwartz function $\phi$ with $\widehat{\phi}$ supported inside $(-1,1)$, we have

$$
\lim _{N \rightarrow \infty} \frac{\sum_{f \in \mathcal{F}_{N}} \mathcal{D}(f, \phi) w_{f}}{\sum_{f \in \mathcal{F}_{N}} w_{f}}=\int_{-\infty}^{\infty} \phi(x) W_{\mathrm{Sp}_{\mathrm{p}}}(x) d x
$$

Here, $N$ ranges over prime values for which $\chi(-N)=1$.

Remark 1.4 The forms $f$ can in fact be taken to range over the family $\mathcal{F}_{N}^{+}$of newforms with epsilon factor $\varepsilon_{f}=1$, since $\Lambda\left(\frac{1}{2}, f\right)=0$ when $\varepsilon_{f}=-1$. The family $\mathcal{F}_{N}^{+}$has symmetry type $\mathrm{SO}$ (even) ( [ILS]). 
The proof is given in Section 6. It uses a special case of the relative trace formula of Ramakrishnan and Rogawski as extended in [FW] by Feigon and Whitehouse. The most general version of their formula (along with the recent improvement [FMP] by File, Martin, and Pitale) could presumably be used to extend the scope of the above theorem.

Theorems 1.1 and 1.3 are derived from weighted equidistribution results for Hecke eigenvalues at a fixed prime $p$, described in more detail below. In each case, the relevant measure is dependent on the value $\chi(p)= \pm 1$. This dependence plays an interesting role in the proof of the above theorems. From the explicit formula, we need to consider the sum over $p$ of the weighted average of the $p$-th Hecke eigenvalue. Because of the nature of the relevant measure, the contribution of the primes satisfying $\chi(p)=1$ differs from that of the primes satisifying $\chi(p)=-1$. We then apply the prime number theorem for arithmetic progressions to get the results.

In general, the Satake parameters of holomorphic modular forms are known to satisfy many equidistribution laws. Foremost is the celebrated Sato-Tate conjecture (proven in [BLGHT]), which asserts that for a fixed non-CM cusp form $f \in S_{k}(N)$, the sequence of normalized Hecke eigenvalues at the unramified primes $p$ (in their natural ordering) is equidistributed in $[-2,2]$ relative to the Sato-Tate measure

$$
d \mu_{\infty}(x)= \begin{cases}\frac{1}{\pi} \sqrt{1-\frac{x^{2}}{4}} d x & \text { if }-2 \leq x \leq 2 \\ 0 & \text { otherwise }\end{cases}
$$

In a different direction, one can fix the prime $p$ and allow the cusp form to vary within a family, possibly with weights. In this setting there are strikingly many different equidistribution results for GL(2) in the literature. ${ }^{2}$ We summarize many of these in Table 1, giving references for the precise statements in each case.

The last of these examples is new. Theorem 3.3 is a generalized and quantitative version of the following. Notation is defined precisely in Section 2.

Theorem 1.5 Let $\chi$ be a primitive real Dirichlet character of conductor $D \geq 1$ coprime to $N$, let $p+D N$ be a fixed prime, and let $\mathcal{F}_{N, k}$ be an orthogonal basis for the space $S_{k}(N)$ of cusp forms, consisting of eigenfunctions of the Hecke operator $T_{p}$, with first Fourier coefficient 1 . Then assuming $N>1$ and $k>2$, the Hecke eigenvalues $\lambda_{f}(p) \epsilon$ $[-2,2]$ for $f \in \mathcal{F}_{N, k}$, when weighted by the central twisted L-values

$$
w_{f}=\frac{\Lambda\left(\frac{1}{2}, f \times \chi\right)}{\|f\|^{2}},
$$

become equidistributed in $[-2,2]$ with respect to the probability measure

$$
d \mu_{p}(x)=\frac{p}{(p+1)-x \chi(p) \sqrt{p}} d \mu_{\infty}(x)
$$

as $N+k \rightarrow \infty$.

\footnotetext{
${ }^{2}$ Some of these have been extended to groups of higher rank, e.g., [Z,BBR, ST], [MT]. There are also some hybrid results for GL(2) with both $p$ and the conductor tending to $\infty$, [Na], [W].
} 


\begin{tabular}{|c|c|c|c|}
\hline Family span & Weights & Measure & References \\
\hline $\begin{array}{l}S_{k}(N) \\
N+k \rightarrow \infty\end{array}$ & 1 & $\begin{array}{l}\text { Plancherel: } \\
\frac{p+1}{\left(p^{1 / 2}+p^{-1 / 2}\right)^{2}-x^{2}} \mu_{\infty}\end{array}$ & {$[\mathrm{Se}, \mathrm{CDF}, \mathrm{Li} 2]$} \\
\hline $\begin{array}{l}L_{0}^{2}\left(\mathrm{SL}_{2}(\mathbf{Z}) \backslash \mathbf{H}\right) \\
\lambda_{j} \leq T^{2}, T \rightarrow \infty\end{array}$ & 1 & Plancherel & {$[\mathrm{Sa}]$} \\
\hline $\begin{array}{l}S_{k}(N) \\
N+k \rightarrow \infty\end{array}$ & $\frac{\left|a_{f}(r)\right|^{2}}{\|f\|^{2}}$ & Sato-Tate $\left(\mu_{\infty}\right)$ & [Li1], [KL2] \\
\hline $\begin{array}{l}L_{0}^{2}\left(\Gamma_{0}(N) \backslash \mathbf{H}\right) \\
N \rightarrow \infty\end{array}$ & $\frac{\left|a_{u_{j}}(r)\right|^{2} h\left(\lambda_{j}\right)}{\left\|u_{j}\right\|^{2}}$ & Sato-Tate & {$[\mathrm{KL} 4]$} \\
\hline $\begin{array}{l}L_{0}^{2}\left(\mathrm{SL}_{2}(\mathbf{Z}) \backslash \mathbf{H}\right) \\
\lambda_{j} \leq T^{2}, T \rightarrow \infty\end{array}$ & $\frac{\left|a_{u_{j}}(r)\right|^{2}}{\left\|u_{j}\right\|^{2}}$ & Sato-Tate & {$[\mathrm{Br}],[\mathrm{BBR}],[\mathrm{BrM}]$} \\
\hline $\begin{array}{l}S_{k}(N)^{\text {new }} \\
N \rightarrow \infty\end{array}$ & $\begin{array}{l}\frac{\Lambda\left(\frac{1}{2}, f \times \chi\right) \Lambda\left(\frac{1}{2}, f\right)}{\|f\|^{2}} \\
\text { for } \chi \text { quadratic }\end{array}$ & $\frac{L_{p}\left(\frac{1}{2}, x, \chi\right) L_{p}\left(\frac{1}{2}, x\right)}{L_{p}(1, \chi)} \mu_{\infty}$ & $\begin{array}{l}\text { [RR1], [FW], } \\
{[\mathrm{SuT}] \text {, and Cor. } 5.2} \\
\text { (Also [Su], [T] for } \\
\text { Maass forms of } \\
\text { increasing level) }\end{array}$ \\
\hline $\begin{array}{l}S_{k}(N) \\
N \rightarrow \infty\end{array}$ & $\begin{array}{l}\text { regular matrix } \\
\text { summation } \\
\text { involving } \frac{1}{\|f\|^{2}}\end{array}$ & $\frac{1}{2}\left(1-\frac{x^{2}}{4}\right)^{-1} \mu_{\infty}$ & [GMR] \\
\hline $\begin{array}{l}S_{k}(N) \\
N+k \rightarrow \infty\end{array}$ & $\frac{\left|a_{f}(r)\right|^{2} \Lambda(s, f \times \chi)}{\|f\|^{2}}$ & $L_{p}(s, x, \chi) \mu_{\infty}$ & Theorem 3.3 below \\
\hline
\end{tabular}

Table 1: Various fixed- $p$ equidistribution results for Hecke eigenvalues on GL(2). (See (2.5) for the definition of $L_{p}(s, x, \chi)$.)

We emphasize that $\chi$ is allowed to be trivial. In the generalized version (Theorem 3.3), $\chi$ need not be real, and we do not specialize the $L$-value in $w_{f}$ to $s=\frac{1}{2}$.

There is a natural interpretation of the measure appearing in the above theorem. See Remark 3.4. Interestingly, the measure is not symmetric, though as expected it converges to the Sato-Tate measure as $p \rightarrow \infty$. It is plotted in Figure 1 in the case $p=5$ when $\chi(5)=1$. If $\chi(p)=-1$, there is an analogous negative bias.

We give another result of this nature in Corollary 5.2, namely that for newforms $f \in S_{k}(N)$ with $N$ prime, the $\lambda_{f}(p)$, when weighted as in Theorem 1.3, become equidistributed in the limit as $N \rightarrow \infty$ relative to the measure $\eta_{p}^{\chi}$ given in the sixth row of Table 1. This is essentially the main result of [RR1]. We obtain a more general statement by keeping track of the dependence on $k$ in their calculations. The measure 


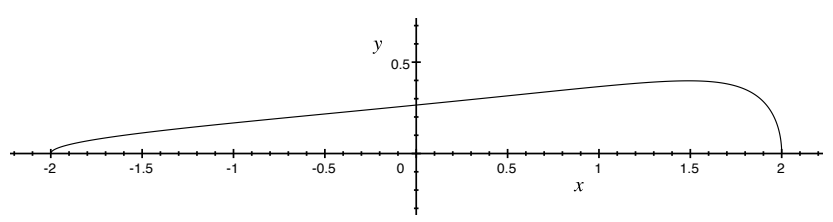

Figure 1

$\eta_{p}^{\chi}$ depends on $\chi$. It exhibits a similar positive bias precisely when $\chi(p)=1$. When $\chi(p)=-1$, it coincides with the Plancherel measure, which is even.

\section{Preliminaries on Modular Forms}

Fix a Dirichlet character $\psi$ modulo $N$, and let $S_{k}(N, \psi)$ be the space of holomorphic cusp forms $f$ on the complex upper half-plane $\mathbf{H}$ that transform under the action of $\Gamma_{0}(N)=\left\{\left(\begin{array}{ll}a & b \\ c & d\end{array}\right) \in \mathrm{SL}_{2}(\mathbf{Z}) \mid c \in N Z\right\}$ according to

$$
f\left(\frac{a z+b}{c z+d}\right)=\psi(d)(c z+d)^{k} f(z) .
$$

We normalize the Petersson inner product on $S_{k}(N, \psi)$ by

$$
\|f\|^{2}=\frac{1}{v(N)} \iint_{\Gamma_{0}(N) \backslash \mathbf{H}}|f(z)|^{2} y^{k} \frac{d x d y}{y^{2}},
$$

where

$$
v(N)=\left[\mathrm{SL}_{2}(\mathbf{Z}): \Gamma_{0}(N)\right] .
$$

For us, a Hecke eigenform is a simultaneous eigenfunction of the Hecke operators

$$
T_{n} f(z)=n^{k-1} \sum_{\substack{a d=n, a>0}} \sum_{b=0}^{d-1} \psi(a) d^{-k} f\left(\frac{a z+b}{d}\right)
$$

for $(n, N)=1$, normalized to have first Fourier coefficient 1 . Given a Hecke eigenform

$$
f(z)=\sum_{n>0} a_{f}(n) q^{n} \quad\left(q=e^{2 \pi i z}\right),
$$

for a prime $p+N$ we fix a complex square root $\psi(p)^{1 / 2}$ and define the normalized $p$-power Hecke eigenvalue

$$
\lambda_{f}\left(p^{\ell}\right)=\frac{a_{f}\left(p^{\ell}\right)}{\psi(p)^{\ell / 2} p^{\ell(k-1) / 2}} \quad(\ell \geq 0) .
$$

By Deligne's theorem, $\lambda_{f}(p) \in[-2,2]$, and our interest is in the distribution of these numbers as $f$ varies. For any integer $\ell \geq 0$,

$$
\lambda_{f}\left(p^{\ell}\right)=X_{\ell}\left(\lambda_{f}(p)\right),
$$


where $X_{\ell}$ is the Chebyshev polynomial of degree $\ell$ defined by $X_{\ell}(2 \cos \theta)=\frac{\sin ((\ell+1) \theta)}{\sin \theta}$ (see, e.g., [KL1, Prop. 29.8], where $\omega^{\prime}$ corresponds to $\psi^{-1}$ ). Equivalently,

$$
a_{f}\left(p^{\ell}\right)=\psi(p)^{\ell / 2} p^{\ell(k-1) / 2} X_{\ell}\left(\lambda_{f}(p)\right) .
$$

Fix an integer $D$ with $(D, N)=1$, and let $\chi$ be a primitive Dirichlet character modulo $D$. The $\chi$-twisted $L$-function of $f$ is given for $\operatorname{Re}(s)>1$ by the Dirichlet series

$$
L(s, f \times \chi)=\sum_{n>0} \frac{\chi(n) a_{f}(n)}{n^{s+\frac{k-1}{2}}} .
$$

The completed $L$-function

$$
\Lambda(s, f \times \chi)=(2 \pi)^{-s-\frac{k-1}{2}} \Gamma\left(s+\frac{k-1}{2}\right) L(s, f \times \chi)
$$

has an analytic continuation to the complex plane and satisfies a functional equation relating $s$ to $1-s$, which takes the form

$$
\Lambda(s, f \times \chi)=\frac{i^{k}}{D^{2 s-1}} \frac{\tau(\chi)^{2}}{D} \Lambda(1-s, f \times \bar{\chi})
$$

when $N=1$. Here, $\tau(\chi)=\sum_{m=1}^{D} \chi(m) e^{2 \pi i m / D}$ is the Gauss sum attached to $\chi$.

Given $x \in[-2,2]$ and $p+D N$, there is a unique unramified unitary representation $\pi_{x, p}$ of $\mathrm{GL}_{2}\left(\mathbf{Q}_{p}\right)$ with Satake parameters $\alpha_{p}, \beta_{p}$ satisfying $\alpha_{p}+\beta_{p}=x \psi(p)^{1 / 2}$ and $\alpha_{p} \beta_{p}=\psi(p)$. We denote its twisted $L$-factor by

$$
L_{p}(s, x, \chi)=\left(1-x \psi(p)^{1 / 2} \chi(p) p^{-s}+\psi(p) \chi(p)^{2} p^{-2 s}\right)^{-1}
$$

With this notation, the local $L$-factor of $L(s, f \times \chi)$ is

$$
L_{p}(s, f \times \chi)=L_{p}\left(s, \lambda_{f}(p), \chi\right) .
$$

\section{Weighted Equidistribution of Hecke Eigenvalues I}

Fix a weight $k>2$ and a level $N>1$, and let

$$
\mathcal{F}=\mathcal{F}_{N, k}=\mathcal{F}_{k}(N, \psi)
$$

be an orthogonal basis for $S_{k}(N, \psi)$ consisting of Hecke eigenforms. Fix $D$ and $\chi$ as above, and fix an integer $r$ relatively prime to $D$. In this section, we do not assume that $\chi^{2}=1$ unless explicitly stated. For each $f \in \mathcal{F}$, define the (complex) weight

$$
w_{f}=\frac{\overline{a_{f}(r)} \Lambda(s, f \times \chi)}{\|f\|^{2}} .
$$

Then for all $s=\sigma+i \tau$ in the strip $1-\frac{k-1}{2}<\sigma<\frac{k-1}{2}$ and all integers $n$ relatively prime to $D N$, by [JK, Theorem 1.1] (which is a twisted version of the main theorem of [KL3]), we have

$$
\begin{aligned}
\frac{1}{v(N)} \sum_{f \in \mathcal{F}} w_{f} a_{f}(n)= & \frac{2^{k-1}(2 \pi r n)^{\frac{k-1}{2}-s}}{(k-2) !} \Gamma\left(s+\frac{k-1}{2}\right) \sum_{d \mid(n, r)} d^{2 s} \psi\left(\frac{n}{d}\right) \chi\left(\frac{r n}{d^{2}}\right) \\
& +O\left(\operatorname{gcd}(n, r) \frac{(4 \pi r n)^{k-1} D^{\frac{k}{2}-\sigma} \varphi(D)}{N^{\sigma+\frac{k-1}{2}}(k-2) !}\right) .
\end{aligned}
$$


(We have adjusted for the fact that in [JK] the $L$-function is normalized to have central point $\frac{k}{2}$, whereas here the central point is $\frac{1}{2}$.) The implied constant is explicit in [JK], and depends only on $s$.

Now fix a prime $p+r N D$. Taking $n=p^{\ell}$ and substituting (2.2), the above becomes

$$
\frac{1}{v(N)} \sum_{f \in \mathcal{F}} w_{f} X_{\ell}\left(\lambda_{f}(p)\right)=F_{\ell}+E_{\ell}
$$

where

$$
F_{\ell}=\left(\psi(p)^{1 / 2} \chi(p) p^{-s}\right)^{\ell} \frac{2^{k-1}(2 \pi r)^{\frac{k-1}{2}-s} \chi(r)}{(k-2) !} \Gamma\left(s+\frac{k-1}{2}\right),
$$

and $E_{\ell}$ is an error term satisfying

$$
E_{\ell} \ll p^{\frac{\ell(k-1)}{2}} \frac{(4 \pi r)^{k-1} D^{\frac{k}{2}-\sigma} \varphi(D)}{N^{\sigma+\frac{k-1}{2}}(k-2) !} .
$$

Proposition 3.1 For any $\ell \geq 0$ and $0<\sigma<1$,

$$
\frac{\sum_{f \in \mathcal{F}_{k}(N, \psi)} w_{f} X_{\ell}\left(\lambda_{f}(p)\right)}{\sum_{f \in \mathcal{F}_{k}(N, \psi)} w_{f}}=\left[\psi(p)^{1 / 2} \chi(p) p^{-s}\right]^{\ell}+O\left(\frac{p^{\frac{\ell(k-1)}{2}}(4 \pi r D e)^{k / 2}}{N^{\frac{k-1}{2}} k^{\frac{k}{2}-1}}\right)
$$

where the implied constant depends only on $r, s, D$.

Remarks $3.2 \quad$ (i) When $N>1$, it is shown in [JK, \$9] that the sum of the weights is nonzero when $N+k$ is sufficiently large. When $N=1$, this can only be verified under certain extra conditions mentioned in Theorem 3.3 below.

(ii) By taking $n=r p^{\ell}$ in (3.2) rather than $n=p^{\ell}$, and using $a_{f}\left(r p^{\ell}\right)=$ $a_{f}(r) a_{f}\left(p^{\ell}\right)$, one obtains (3.3) with the different weight

$$
w_{f}=\frac{\Lambda(s, f \times \chi)\left|a_{f}(r)\right|^{2}}{\|f\|^{2}} .
$$

In (3.4) we then have to replace $(2 \pi r)$ by $\left(2 \pi r^{2}\right)$, and $\chi(r)$ by $\sum_{d \mid r} d^{2 s} \psi\left(\frac{r}{d}\right) \chi\left(\frac{r^{2}}{d^{2}}\right)$; in (3.5), $r^{2}$ replaces $r$, and one additional factor of $r$ is needed due to $\operatorname{gcd}(n, r)=r$. As long as the above sum over $d$ is nonzero (for example, if $\chi^{2}$ and $\psi$ are trivial and $s$ is real), (3.6) holds with the alternative weight upon replacing $r$ by $r^{2}$ in the error term.

Proof of Proposition 3.1 In the notation of (3.3), the left-hand side of (3.6) is

$$
\frac{F_{\ell}+E_{\ell}}{F_{0}+E_{0}}=\frac{F_{\ell}}{F_{0}}+\frac{E_{\ell}-\frac{F_{\ell}}{F_{0}} E_{0}}{F_{0}+E_{0}} .
$$

This will immediately give (3.6) once we show that the second term on the righthand side has the desired rate of decay. If we denote the right-hand side of (3.5) by 
$p^{\ell(k-1) / 2} C_{0}$, then

$$
\begin{aligned}
\frac{E_{\ell}-\frac{F_{\ell}}{F_{0}} E_{0}}{F_{0}+E_{0}} & =\frac{E_{\ell}-\psi(p)^{\ell / 2} \chi(p)^{\ell} p^{-\ell s} E_{0}}{F_{0}+E_{0}} \ll \frac{\left(p^{\frac{\ell(k-1)}{2}}+p^{-\ell \sigma}\right) C_{0}}{F_{0}+E_{0}} \\
& \ll \frac{p^{\frac{\ell(k-1)}{2}} C_{0}}{F_{0}+E_{0}}=p^{\frac{\ell(k-1)}{2}} \frac{\frac{C_{0}}{F_{0}}}{1+\frac{E_{0}}{F_{0}}} .
\end{aligned}
$$

In [JK, §9] (taking $n=1$ ), it is shown that

$$
\frac{E_{0}}{F_{0}} \ll \frac{C_{0}}{F_{0}} \ll \frac{(4 \pi r D e)^{k / 2}}{N^{(k-1) / 2} k^{k / 2-1}},
$$

where the implied constant depends on $r, s, D$. The proposition follows.

Define a measure

$$
d \mu_{p, s, \chi}(x)=\sum_{\ell=0}^{\infty}\left[\psi(p)^{1 / 2} \chi(p) p^{-s}\right]^{\ell} X_{\ell}(x) d \mu_{\infty}(x),
$$

where, as before, $\mu_{\infty}$ is the Sato-Tate measure on $\mathbf{R}$ with support $[-2,2]$, and $X_{\ell}$ is the Chebyshev polynomial. The infinite series is absolutely convergent provided $|x| \leq 2$ and $\operatorname{Re}(s)>0$. Indeed, if $|x| \leq 2$ and $|t|<1$, we have the well-known identity

$$
\sum_{\ell=0}^{\infty} t^{\ell} X_{\ell}(x)=\frac{1}{1-x t+t^{2}} \text {. }
$$

Therefore

$$
d \mu_{p, s, \chi}(x)=\frac{1}{1-x \psi(p)^{1 / 2} \chi(p) p^{-s}+\psi(p) \chi(p)^{2} p^{-2 s}} d \mu_{\infty}(x) .
$$

As pointed out to us by Fan Zhou, this gives (in the notation of (2.5))

$$
d \mu_{p, s, \chi}(x)=L_{p}(s, x, \chi) d \mu_{\infty}(x) .
$$

The above is a complex-valued probability measure, since, by (3.7) and the orthonormality of the $X_{\ell}(x)$ relative to $\mu_{\infty}, \int X_{0}(x) d \mu_{p, s, \chi}=1$. We note that when $s=\frac{1}{2}, \psi$ is trivial, $\psi(p)^{1 / 2}$ is chosen to be 1 , and $\chi$ is real;

$$
d \mu_{p, \frac{1}{2}, \chi}(x)=\frac{p}{(p+1)-x \chi(p) \sqrt{p}} d \mu_{\infty}(x)
$$

is the measure given in Theorem 1.5 .

Theorem 3.3 Fix $s$ in the critical strip $0<\operatorname{Re}(s)<1$, let $N>1$ be coprime to $r D$, let $k>2$, let $\psi$ be a Dirichlet character whose conductor divides $N$, fix a prime $p+r N D$, and a choice of square root $\psi(p)^{1 / 2}$. Define weights $w_{f}$ as in (3.1) and Hecke eigenvalues $\lambda_{f}(p)$ as in (2.1). Then the $\lambda_{f}(p)$ for $f \in \mathcal{F}_{k}(N, \psi)$ become $w_{f}$-equidistributed in $[-2,2]$ relative to the measure $\mu_{p, s, \chi}$ as $N+k \rightarrow \infty$. In other words, for any continuous function $\phi$ on $\mathbf{R}$,

$$
\lim _{N+k \rightarrow \infty} \frac{\sum_{f \in \mathcal{F}_{k}(N, \psi)} w_{f} \phi\left(\lambda_{f}(p)\right)}{\sum_{f \in \mathcal{F}_{k}(N, \psi)} w_{f}}=\int_{\mathbf{R}} \phi d \mu_{p, s, \chi} .
$$


Moreover, if $\phi$ is a polynomial of degree $d$, then

$$
\frac{\sum_{f \in \mathcal{F}_{k}(N, \psi)} w_{f} \phi\left(\lambda_{f}(p)\right)}{\sum_{f \in \mathcal{F}_{k}(N, \psi)} w_{f}}=\int_{\mathbf{R}} \phi d \mu_{p, s, \chi}+O\left(\frac{p^{\frac{d(k-1)}{2}}(4 \pi r D e)^{k / 2}}{N^{\frac{k-1}{2}} k^{\frac{k}{2}-1}}\|\phi\|_{S T}\right),
$$

where $\|\phi\|_{S T}$ is the norm of $\phi$ in $L^{2}\left(\mathbf{R}, \mu_{\infty}\right)$.

When $N=1$, the equidistribution assertion (3.10) still holds, provided $\chi^{2}=1, s=\frac{1}{2}$ and $i^{k} \tau(\chi)^{2} / D \neq-1$.

Lastly, if $\chi$ is quadratic, $\psi$ is trivial, and $s$ is real, then all of the above statements hold if instead we use the nonnegative weights given in (1.1) and we replace $r$ by $r^{2}$ in the error term of (3.11).

Remark 3.4 The measure $\mu_{p, s, \chi}$ appearing here is natural for the following reason. The weight $w_{f}$ depends directly on $\lambda_{f}(p)$ via the local $L$-factor $L_{p}(s, f \times \chi)=$ $L_{p}\left(s, \lambda_{f}(p), \chi\right)$ (in the notation of $(2.5)$ ). Assuming the remaining $L$-factors do not affect the distribution of the $\lambda_{f}(p)$, on the left-hand side of (3.10) we have something resembling a Petersson-weighted average of the function $L_{p}(s, x, \chi) \phi(x)$ at the points $\lambda_{f}(p)$, which, in view of the equidistribution result [Lil], tends to the integral of this function against the Sato-Tate measure. By (3.9), this is exactly what appears on the right-hand side of (3.10).

Proof First take $N>1$. By the fact that the Chebyshev polynomials are orthonormal relative to the Sato-Tate measure $\mu_{\infty}$, we see from (3.7) that (3.6) gives (3.10) with $\phi=X_{\ell}$ for $\ell \geq 0$. By linearity it holds if $\phi$ is any polynomial, so by Weierstrass approximation, (3.10) holds for all continuous functions.

Since $\left\|X_{\ell}\right\|_{s T}=1$ for all $\ell$, Proposition 3.1 gives (3.11) when $\phi=X_{\ell}$. For an arbitrary polynomial $\phi$ of degree $d$, we can write $\phi=\sum_{\ell=0}^{d}\left\langle\phi, X_{\ell}\right\rangle X_{\ell}$, so denoting the left-hand side of (3.11) by $\mathcal{E}(\phi)$, we have

$$
\left|\mathcal{E}(\phi)-\int \phi d \mu_{p, s, \chi}\right|=\left|\sum_{\ell=0}^{d}\left\langle\phi, X_{\ell}\right\rangle\left(\mathcal{E}\left(X_{\ell}\right)-\int X_{\ell} d \mu_{p, s, \chi}\right)\right| .
$$

Applying (3.7), (3.6), and the Schwarz inequality $\left|\left\langle\phi, X_{\ell}\right\rangle\right| \leq\|\phi\|_{S T}$, the above is

$$
\ll\|\phi\|_{S T} \frac{(4 \pi r D e)^{k / 2}}{N^{\frac{k-1}{2}} k^{\frac{k}{2}-1}} \sum_{\ell=0}^{d} p^{\frac{\ell(k-1)}{2}}
$$

and (3.11) follows.

Now suppose $N=1, \chi^{2}=1$, and $s=\frac{1}{2}$. Then there is an extra main term in [JK, Theorem 1.1], so that in place of (3.4), we have

$$
F_{\ell}=\left(\chi(p) p^{-1 / 2}\right)^{\ell} \frac{2^{k-1}(2 \pi r)^{\frac{k}{2}-1} \chi(r)}{(k-2) !} \Gamma\left(\frac{k}{2}\right)\left[1+i^{k} \frac{\tau(\chi)^{2}}{D}\right] .
$$

(The extra main term contains the factor $\overline{\chi\left(r p^{\ell}\right)}$, so we we have imposed $\chi^{2}=1$ to make this equal to $\chi(p)^{\ell} \chi(r)$.) The rest of the argument then goes through as above, provided the bracketed expression is nonzero.

Finally, if the alternative nonnegative weights (1.1) are used, then in view of Remark 3.2(ii), everything goes through as above. 


\section{Low-lying Zeros I}

In this section we derive Theorem 1.1 from the results of the previous section by standard methods (see, for example, [Ko, \$9]). We will use Proposition 3.1, together with the following consequence of the explicit formula for the $L$-function of a holomorphic newform $f \in \mathcal{F}_{k}(N)^{\text {new }}$ with analytic conductor $Q_{f}=k^{2} N$ :

$$
\begin{aligned}
\mathcal{D}(f, \phi)=\widehat{\phi}(0)+\frac{1}{2} \phi(0)-2 \sum_{p \nmid N} \frac{\lambda_{f}(p) \log p}{p^{1 / 2} \log Q_{f}} \widehat{\phi}\left(\frac{\log p}{\log Q_{f}}\right) \\
-2 \sum_{p \nmid N} \frac{\lambda_{f}\left(p^{2}\right) \log p}{p \log Q_{f}} \widehat{\phi}\left(\frac{2 \log p}{\log Q_{f}}\right)+O\left(\frac{\log \log 3 N}{\log Q_{f}}\right) .
\end{aligned}
$$

This holds for any even Schwartz function $\phi$ on $\mathbf{R}$ whose Fourier transform has compact support, [ILS, Lemma 4.1].

For the remainder of this section, $\chi$ is a real Dirichlet character, and $\mathcal{F}$ denotes one of the following families given in Theorem 1.1:

(a) $\mathcal{F}=\mathcal{F}_{k}(1)$, the set of Hecke eigenforms of level $N=1$ and even weight $k$ chosen so that $\frac{i^{k} \tau(\chi)^{2}}{D} \neq-1$.

(b) $\mathcal{F}=\mathcal{F}_{k}(N)^{\text {new }}$, where $N+r D$ is prime. In this case, the even weight $k \geq 4$ is chosen so that $\frac{i^{k} \tau(\chi)^{2}}{D}=-1$.

We need to consider the weighted average of $\mathcal{D}(f, \phi)$ over $\mathcal{F}$. To simplify notation, given a function $A$ : $f \mapsto A_{f}$ on $\mathcal{F}$, we define the $w$-weighted average of $A$ by

$$
\mathcal{E}_{\mathcal{F}}^{w}(A)=\frac{\sum_{f \in \mathcal{F}} A_{f} w_{f}}{\sum_{f \in \mathcal{F}} w_{f}},
$$

where, for all $f$ we take $w_{f}$ to be either the weight defined in (3.1) with $s=\frac{1}{2}$, or the weight defined in (1.1). In the latter case, Remark 3.2(ii) should be borne in mind for the remainder of this section.

When $N=1$ and $\frac{i^{k} \tau(\chi)^{2}}{D}=-1$, or equivalently, $\chi(-1)=-i^{k}$, the functional equation (2.4) forces $\Lambda\left(\frac{1}{2}, f \times \chi\right)=0$ since $\chi$ is real. Hence when $N$ is prime, the conditions imposed on $k$ and $\chi$ ensure that $w_{f}=0$ for all Hecke eigenforms $f$ of level 1 and weight $k$. If we set $f_{N}(z)=f(N z)$ for such $f$, we have

$$
\Lambda\left(\frac{1}{2}, f_{N} \times \chi\right)=\frac{\chi(N)}{N^{k / 2}} \Lambda\left(\frac{1}{2}, f \times \chi\right)=0
$$

as well, so that $w_{h}=0$ for all $h$ in the span of $\left\{f, f_{N}\right\}$. Therefore,

$$
\mathcal{E}_{\mathcal{F}}^{w}(A)=\mathcal{E}_{\mathcal{F}_{k}(N)}^{w}(A)
$$

in this case; i.e., the value is unaffected if we average over an orthogonal basis for the full space $S_{k}(N)$, rather than restricting to newforms. 
Since $Q_{f}=k^{2} N$ is constant across $\mathcal{F}$, we denote it by $Q$ in what follows. By (4.1), we have

$$
\begin{aligned}
\frac{\sum_{f \in \mathcal{F}} \mathcal{D}(f, \phi) w_{f}}{\sum_{f \in \mathcal{F}} w_{f}}= & \widehat{\phi}(0)+\frac{1}{2} \phi(0)+O\left(\frac{\log \log 3 N}{\log Q}\right) \\
& -2 \sum_{p \nmid N} \frac{\mathcal{E}_{\mathcal{F}}^{w}(\lambda \cdot(p)) \log p}{p^{1 / 2} \log Q} \widehat{\phi}\left(\frac{\log p}{\log Q}\right) \\
& -2 \sum_{p \nmid N} \frac{\mathcal{E}_{\mathcal{F}}^{w}\left(\lambda \cdot\left(p^{2}\right)\right) \log p}{p \log Q} \widehat{\phi}\left(\frac{2 \log p}{\log Q}\right) .
\end{aligned}
$$

Taking $s=\frac{1}{2}, \psi$ trivial, and $\ell=1,2$ in (3.6), we have (using (4.2) when $N$ is prime)

$$
\begin{gathered}
\mathcal{E}_{\mathcal{F}}^{w}(\lambda .(p))=\chi(p) p^{-1 / 2}+O\left(\frac{p^{\frac{k-1}{2}} R^{k}}{N^{\frac{k-1}{2}} k^{\frac{k}{2}-1}}\right), \\
\mathcal{E}_{\mathcal{F}}^{w}\left(\lambda .\left(p^{2}\right)\right)=\chi(p)^{2} p^{-1}+O\left(\frac{p^{k-1} R^{k}}{N^{\frac{k-1}{2}} k^{\frac{k}{2}-1}}\right)
\end{gathered}
$$

for a positive constant $R$ depending on $D$ and $r$. It is a consequence of the prime number theorem that for any real number $m>-1$,

$$
\sum_{p \leq x} p^{m} \log p \sim \frac{x^{m+1}}{m+1}
$$

as $x \rightarrow \infty$. If the support of $\widehat{\phi}$ is contained in $[-\alpha, \alpha]$, the sum in (4.3) is restricted to $p \leq Q^{\alpha}$. Therefore, the contribution to (4.3) of the error term in (4.5) is

$$
\ll \frac{R^{k}}{N^{\frac{k-1}{2}} k^{\frac{k}{2}-1}} \sum_{p \leq Q^{\alpha}} p^{\frac{k}{2}-1} \log p \ll \frac{Q^{\frac{\alpha k}{2}} R^{k}}{\left(\frac{k}{2}\right) N^{\frac{k-1}{2}} k^{\frac{k}{2}-1}}=\frac{2 N^{\frac{\alpha k}{2}} k^{\alpha k} R^{k}}{N^{\frac{k-1}{2}} k^{\frac{k}{2}}}=O\left(\frac{1}{\log Q}\right),
$$

provided $\alpha<\frac{1}{2}$. (If $k$ is fixed, we only need $\alpha<1-\frac{1}{k}$.) The contribution to (4.4) of the error term in (4.6) is

$$
\ll \frac{R^{k}}{N^{\frac{k-1}{2}} k^{\frac{k}{2}-1}} \sum_{p \leq Q^{\alpha / 2}} p^{k-2} \log p \ll \frac{Q^{\frac{\alpha(k-1)}{2}} R^{k}}{(k-1) N^{\frac{k-1}{2}} k^{\frac{k}{2}-1}} \ll \frac{N^{\frac{\alpha(k-1)}{2}} k^{\alpha(k-1)} R^{k}}{N^{\frac{k-1}{2}} k^{\frac{k}{2}}},
$$

which may likewise be absorbed into the error term if $\alpha<\frac{1}{2}$.

It remains to treat the contribution of the main terms of (4.5) and (4.6) to (4.3) and (4.4), respectively. If $\chi$ is trivial, the former yields

$$
-2 \sum_{p+N} \frac{\log p}{p \log Q} \widehat{\phi}\left(\frac{\log p}{\log Q}\right)=-2 \sum_{p} \frac{\log p}{p \log Q} \widehat{\phi}\left(\frac{\log p}{\log Q}\right)+O\left(\frac{\log \log 3 N}{\log Q}\right)
$$

(by $\left(4.19^{\prime}\right)$ of [ILS]), which in turn is

$$
=-\phi(0)+O\left(\frac{\log \log 3 N}{\log Q}\right)
$$

by the prime number theorem, using the fact that $\phi$ is even. 
On the other hand, if $\chi$ is nontrivial, then the main term of (4.5) contributes

$$
-2 \sum_{p: \chi(p)=1} \frac{\log p}{p \log Q} \widehat{\phi}\left(\frac{\log p}{\log Q}\right)+2 \sum_{p: \chi(p)=-1} \frac{\log p}{p \log Q} \widehat{\phi}\left(\frac{\log p}{\log Q}\right)+O\left(\frac{\log \log 3 N}{\log Q}\right) \text {. }
$$

The value of $\chi$ is 1 on exactly half of the primes. By the prime number theorem for arithmetic progressions, the above is

$$
=-\frac{1}{2} \phi(0)+\frac{1}{2} \phi(0)+O\left(\frac{\log \log 3 N}{\log Q}\right)=O\left(\frac{\log \log 3 N}{\log Q}\right) .
$$

Lastly, for any real $\chi$, the contribution of the main term of (4.6) is

$$
\ll 2 \sum_{p+N} \frac{\log p}{p^{2} \log Q}\left|\widehat{\phi}\left(\frac{2 \log p}{\log Q}\right)\right|=O\left(\frac{1}{\log Q}\right) .
$$

Putting everything together, we conclude that when $\alpha<\frac{1}{2}$,

$$
\frac{\sum_{f \in \mathcal{F}} \mathcal{D}(f, \phi) w_{f}}{\sum_{f \in \mathcal{F}} w_{f}}= \begin{cases}\widehat{\phi}(0)-\frac{1}{2} \phi(0)+O\left(\frac{\log \log 3 N}{\log \left(k^{2} N\right)}\right), & \chi \text { trivial } \\ \widehat{\phi}(0)+\frac{1}{2} \phi(0)+O\left(\frac{\log \log 3 N}{\log \left(k^{2} N\right)}\right), & \chi \text { nontrivial }\end{cases}
$$

which proves Theorem 1.1.

\section{Weighted Equidistribution of Hecke Eigenvalues II}

We recall the setup from Theorem 1.3: $-D<0$ is the discriminant of a quadratic field $E=\mathbf{Q}[\sqrt{-D}], \chi=\chi_{-D}$ is the associated primitive quadratic character modulo $D$ given by the Kronecker symbol $n \mapsto\left(\frac{-D}{n}\right)$, and $N$ is a prime number for which $\chi(-N)=1$. The latter condition means that $N$ is inert in $E$. For $k>2$ even, we let $\mathcal{F}=\mathcal{F}_{N, k}^{\text {new }}$ be the set of holomorphic newforms of weight $k$ and level $N$. For $f \in \mathcal{F}$, we define the weight

$$
w_{f}=\frac{\Lambda\left(\frac{1}{2}, f \times \chi\right) \Lambda\left(\frac{1}{2}, f\right)}{\|f\|^{2}}=\frac{\Lambda\left(\frac{1}{2}, f_{E}\right)}{\|f\|^{2}}
$$

where $f_{E}$ is the base change of $f$ to $E$.

Proposition 5.1 With hypotheses as above, for any $\ell \geq 0$, and any prime $p+N D$,

$$
\mathcal{E}_{\mathcal{F}}^{w}\left(\lambda .\left(p^{\ell}\right)\right):=\frac{\sum_{f \in \mathcal{F}} w_{f} X_{\ell}\left(\lambda_{f}(p)\right)}{\sum_{f \in \mathcal{F}} w_{f}}=\int_{\mathbf{R}} X_{\ell} d \eta_{p}^{\chi}+O\left(\frac{p^{\ell\left(k+\frac{1}{2}\right)} D^{k}}{k^{1 / 2} N^{k / 2-\varepsilon}}\right),
$$

where

$$
\eta_{p}^{\chi}(x)= \begin{cases}\frac{p+1}{\left(p^{1 / 2}+p^{-1 / 2}\right)^{2}-x^{2}} \mu_{\infty}(x) & \text { if } \chi(p)=-1, \\ \frac{p-1}{\left(p^{1 / 2}+p^{-1 / 2}-x\right)^{2}} \mu_{\infty}(x) & \text { if } \chi(p)=1,\end{cases}
$$

and the implied constant depends only on $\chi, \ell, D$, and $\varepsilon \in(0,1)$. Furthermore, if $N>$ $p^{\ell} D$, then (5.1) holds with no error term:

$$
\mathcal{E}_{\mathcal{F}}^{w}\left(\lambda \cdot\left(p^{\ell}\right)\right)=\int_{\mathbf{R}} X_{\ell} d \eta_{p}^{\chi}
$$


Equation (5.1) is essentially the main result of [RR1], but we have divided by the sum of the weights, and shown the dependence on $p$ and $k$ explicitly in the error term. The proof is somewhat involved, so we defer it to Section 7.2. Equation (5.2) likewise follows from a special case of [FW, Theorem 6.1]. Details are provided in Section 7.1.

Corollary 5.2 Assume the hypotheses above. Then the multiset $\left\{\lambda_{f}(p) \mid f \in \mathcal{F}_{N, k}^{\text {new }}\right\}$ of normalized $p$-th Hecke eigenvalues, when weighted as above, becomes equidistributed in $[-2,2]$ with respect to the measure $\eta_{p}^{\chi}$ as $N \rightarrow \infty$. Thus, for any continuous function $\phi$,

$$
\lim _{N \rightarrow \infty} \frac{\sum_{f \in \mathcal{F}_{N, k}^{\text {new }}} w_{f} \phi\left(\lambda_{f}(p)\right)}{\sum_{f \in \mathcal{F}_{N, k}^{\text {new }}} w_{f}}=\int_{\mathbf{R}} \phi d \eta_{p}^{\chi} .
$$

Moreover, if $\phi$ is a polynomial of degree $d$, then

$$
\frac{\sum_{f \in \mathcal{F}} w_{f} \phi\left(\lambda_{f}(p)\right)}{\sum_{f \in \mathcal{F}} w_{f}}=\int_{\mathbf{R}} \phi d \eta_{p}^{\chi}+O\left(\|\phi\|_{S T} \frac{p^{d\left(k+\frac{1}{2}\right)} D^{k}}{k^{1 / 2} N^{k / 2-\varepsilon}}\right) .
$$

The error term in (5.4) vanishes if $N>p^{d} D$.

Remarks $5.3 \quad$ (i) In [RR1, Theorem A], a much stronger claim is made, namely that in (5.4), by Weierstrass approximation we can take $\phi$ to be the characteristic function of any subinterval of $[-2,2]$, preserving the error term $O\left(N^{-k / 2+\varepsilon}\right)$. However, because the error in (5.4) depends in a crucial way on the approximating polynomial $\phi$, their argument is incomplete. Possibly one could use the method of Murty and Sinha [MS], but we have not investigated this.

(ii) Because of (5.2), the weight $k$ can vary in any fashion as $N \rightarrow \infty$. However we cannot obtain the conclusion for fixed $N$ and $k \rightarrow \infty$, because the factor $\left(p^{\ell} D / N\right)^{k / 2}$ in the error term of (5.1) will tend to $\infty$ rapidly with $k$ when $\ell$ is large.

(iii) It is not hard to show that

$$
\eta_{p}^{\chi}(x)=\frac{L_{p}\left(\frac{1}{2}, x, \chi\right) L_{p}\left(\frac{1}{2}, x\right)}{L_{p}(1, \chi)} \mu_{\infty}(x),
$$

in the notation of (2.5). So the above result can be interpreted in a manner analogous to the remark after Theorem 3.3.

Proof of Corollary 5.2 The limit (5.3) holds for $\phi=X_{\ell}$ by (5.1), and then Weierstrass approximation gives it for any continuous $\phi$. The rest of the proof proceeds in just the same way as that of Theorem 3.3.

\section{Low-lying Zeros II}

Here we will use Proposition 5.1 to prove Theorem 1.3. First we need to compute the integrals of the Chebyshev polynomials against the measure $\eta_{p}^{\chi}$ defined in Proposition 5.1. 
Proposition 6.1 Let $r \geq 0$ be an integer. Then if $\chi(p)=-1$,

$$
\int_{-\infty}^{\infty} X_{r} d \eta_{p}^{\chi}= \begin{cases}p^{-r / 2} & \text { if } r \text { is even } \\ 0 & \text { if } r \text { is odd }\end{cases}
$$

If $\chi(p)=1$, then

$$
\int_{-\infty}^{\infty} X_{r} d \eta_{p}^{\chi}=(r+1) p^{-r / 2}
$$

Proof The first assertion is well known ( [Se, p. 79]). For the second, using (3.8) we have

$$
\begin{aligned}
& \frac{p-1}{\left(p^{1 / 2}+p^{-1 / 2}-x\right)^{2}} \\
& \quad=\frac{1-\frac{1}{p}}{\left(1-p^{-1 / 2} x+p^{-1}\right)^{2}}=\left(1-\frac{1}{p}\right)\left[\sum_{n=0}^{\infty} p^{-n / 2} X_{n}(x)\right]^{2} \\
& =\left(1-\frac{1}{p}\right)\left[\sum_{j=0}^{\infty} X_{j}(x)^{2} p^{-j}+2 \sum_{m=1}^{\infty} \sum_{n=0}^{m-1} X_{m}(x) X_{n}(x) p^{-(m+n) / 2}\right] .
\end{aligned}
$$

By the Clebsch-Gordon formula (or by induction via $X_{n+1}(x)=x X_{n}(x)-X_{n-1}(x)$ ), we have

$$
X_{m}(x) X_{n}(x)=\sum_{k=0}^{n} X_{m-n+2 k}(x), \quad(n \leq m) .
$$

So (6.1) becomes

$$
\left(1-\frac{1}{p}\right)\left[\sum_{j=0}^{\infty} \sum_{t=0}^{j} X_{2 t}(x) p^{-j}+2 \sum_{m=1}^{\infty} \sum_{n=0}^{m-1} \sum_{k=0}^{n} X_{m-n+2 k}(x) p^{-(m+n) / 2}\right] .
$$

For the double sum,

$$
\sum_{j=0}^{\infty} \sum_{t=0}^{j} X_{2 t}(x) p^{-j}=\sum_{t=0}^{\infty} X_{2 t}(x) \sum_{j=0}^{\infty} p^{-(j+t)}=\left(1-\frac{1}{p}\right)^{-1} \sum_{t=0}^{\infty} X_{2 t}(x) p^{-t}
$$

For the triple sum, we observe that the map $(m, n, k) \mapsto(m-n+2 k, m-n, m)$ defines a bijection from

$$
\{(m, n, k) \mid m \geq 1,0 \leq n \leq m-1,0 \leq k \leq n\}
$$

to

$$
\left\{(u, b, m) \mid u \geq 1,1 \leq b \leq u, b \equiv u \bmod 2, m \geq \frac{u+b}{2}\right\}
$$


with inverse $(u, b, m) \mapsto\left(m, m-b, \frac{u-b}{2}\right)$. Therefore,

$$
\begin{aligned}
\sum_{m=1}^{\infty} \sum_{n=0}^{m-1} \sum_{k=0}^{n} X_{m-n+2 k}(x) p^{-(m+n) / 2} & \\
= & \sum_{u=1}^{\infty} X_{u}(x) \sum_{\substack{b \equiv u \bmod 2 \\
1 \leq b \leq u}} \sum_{m=\frac{u+b}{2}}^{\infty} p^{-(2 m-b) / 2} \\
& =\sum_{u=1}^{\infty} X_{u}(x) \sum_{\substack{b \equiv u \bmod 2 \\
1 \leq b \leq u}} p^{b / 2} p^{-(u+b) / 2}\left(1-\frac{1}{p}\right)^{-1} \\
& =\left(1-\frac{1}{p}\right)^{-1} \sum_{u=1}^{\infty} X_{u}(x) p^{-u / 2} \sum_{\substack{u=u \bmod 2 \\
1 \leq b \leq u}} 1 .
\end{aligned}
$$

The sum over $b$ has the value $\frac{u}{2}$ if $u$ is even, and $\frac{u+1}{2}$ if $u$ is odd. Using this and (6.3), (6.2) becomes

$$
\sum_{r \geq 0 \text { even }} X_{r}(x) p^{-r / 2}+2 \sum_{r \geq 2 \text { even }} \frac{r}{2} X_{r}(x) p^{-r / 2}+2 \sum_{r \geq 1 \text { odd }} \frac{r+1}{2} X_{r}(u) p^{-r / 2} .
$$

In the middle sum, we can actually take $r \geq 0$ because of the $\frac{r}{2}$ coefficient. This proves that

$$
d \eta_{p}^{\chi}(x)=\sum_{r=0}^{\infty}(r+1) p^{-r / 2} X_{r}(x) d \mu_{\infty}(x) .
$$

The proposition now follows immediately using the orthonormality of the Chebyshev polynomials relative to $d \mu_{\infty}$.

With this proposition in hand, we obtain the following two special cases of Proposition 5.1.

Corollary 6.2 In the notation of Proposition 5.1, for any $0<\varepsilon<1$,

$$
\mathcal{E}_{\mathcal{F}}^{w}(\lambda .(p))= \begin{cases}2 p^{-1 / 2}+O\left(\frac{p^{k+\frac{1}{2}}}{N^{k / 2-\varepsilon}}\right) & \text { if } \chi(p)=1, \\ O\left(\frac{p^{k+\frac{1}{2}}}{N^{k / 2-\varepsilon}}\right) & \text { if } \chi(p)=-1\end{cases}
$$

the error terms vanishing if $p<\frac{N}{D}$, and

$$
\mathcal{E}_{\mathcal{F}}^{w}\left(\lambda .\left(p^{2}\right)\right)= \begin{cases}3 p^{-1}+O\left(\frac{p^{2 k+1}}{N^{k / 2-\varepsilon}}\right) & \text { if } \chi(p)=1, \\ p^{-1}+O\left(\frac{p^{2 k+1}}{N^{k / 2-\varepsilon}}\right) & \text { if } \chi(p)=-1,\end{cases}
$$

the error terms vanishing if $p^{2}<\frac{N}{D}$. Implied constants depend on $k, D$, and $\varepsilon$.

We can now prove Theorem 1.3 following the method in Section 4. Suppose that $\operatorname{Supp}(\phi) \subseteq[-\alpha, \alpha]$ for some $\alpha<1$. Then for all $N$ sufficiently large,

$$
Q^{\alpha}=N^{\alpha} k^{2 \alpha}<\frac{N}{D} \text {. }
$$


In the explicit formula, the sum (4.3) involves only primes $p \leq Q^{\alpha}$, which by the above means that (6.4) holds with no error term. Therefore, (4.3) is equal to

$$
-2 \sum_{\substack{p+N \\ \chi(p)=1}} \frac{2 \log p}{p \log Q} \widehat{\phi}\left(\frac{\log p}{\log Q}\right)=-4 \sum_{\substack{p \\ \chi(p)=1}} \frac{\log p}{p \log Q} \widehat{\phi}\left(\frac{\log p}{\log Q}\right)+O\left(\frac{\log \log 3 N}{\log Q}\right) .
$$

Because $\chi$ is a nontrivial quadratic character, its value is 1 on exactly half of the primes. By the prime number theorem for arithmetic progressions, the above is

$$
=-\phi(0)+O\left(\frac{\log \log 3 N}{\log Q}\right) \text {. }
$$

The sum (4.4) involves only primes satisfying $p^{2} \leq Q^{\alpha}$. So for sufficiently large $N$ as above, we may apply (6.5) with no error term. Substituting it into (4.4), one obtains an expression that can be absorbed into the error term $O(\log \log 3 N / \log Q)$, as in (4.7).

It now follows that if $\alpha<1$ and $N$ satisfies (6.6),

$$
\frac{\sum_{f \in \mathcal{F}} \mathcal{D}(f, \phi) w_{f}}{\sum_{f \in \mathcal{F}} w_{f}}=\widehat{\phi}(0)-\frac{1}{2} \phi(0)+O\left(\frac{\log \log 3 N}{\log N}\right)
$$

for an implied constant depending only on $\phi$. This proves Theorem 1.3.

We remark that if we instead fix $N$ and allow $k \rightarrow \infty$, we cannot obtain the analog of Theorem 1.3 by this method. Indeed, the contribution of the error term in (6.4) to (4.3) gives an expression involving

$$
\sum_{p \leq Q^{\alpha}} p^{k} \log p
$$

which up to small powers of $k$ grows like $k^{\alpha k}$. There is not enough decay in the $k$ aspect in (5.1) to cancel this growth as $k \rightarrow \infty$ for any $\alpha>0$.

\section{Proof of Proposition 5.1}

The papers [RR1, FW] each use the relative trace formula to develop a formula for an average of $L$-values, which in the simplest case takes the form

$$
\sum_{f \in \mathcal{F}_{N, k}^{\text {new }}} w_{f} \widehat{f}_{p}\left(\pi_{p}\right)
$$

where: $w_{f}=\Lambda\left(\frac{1}{2}, f \times \chi\right) \Lambda\left(\frac{1}{2}, f\right) /\|f\|^{2}$ for a quadratic character $\chi=\chi_{-D}, N$ is a prime not dividing $D, \chi(-N)=1, k>2$ is even, $\widehat{f}_{p}$ is the Satake transform of a compactly supported bi-GL $\mathrm{GL}_{2}\left(\mathbf{Z}_{p}\right)$-invariant local test function $f_{p}: \mathrm{GL}_{2}\left(\mathbf{Q}_{p}\right) \rightarrow \mathbf{C}$, and $\pi_{p}$ is the unramified local representation determined by the cusp form $f$. (In [RR1] the notation $f_{p}^{\wedge}\left(a_{p}(\varphi)\right)$ is used, where $a_{p}(\varphi)$ corresponds to our $\lambda_{f}(p)$.)

For our purpose, we need to choose the particular test function $f_{p}$ whose Satake transform is equal to the Chebyshev polynomial $X_{\ell}$. This function is given as follows. 
For $K_{p}=\mathrm{GL}_{2}\left(\mathbf{Z}_{p}\right)$ and $Z_{p}$ the center of $\mathrm{GL}_{2}\left(\mathbf{Q}_{p}\right)$, let

$$
\begin{aligned}
M\left(p^{\ell}\right) & =\bigcup_{\substack{i+j=\ell \\
i \geq j \geq 0}} Z_{p} K_{p}\left(\begin{array}{ll}
p^{i} & \\
& p^{j}
\end{array}\right) K_{p}=\bigcup_{\substack{i+j=\ell \\
i \geq j \geq 0}} Z_{p} K_{p}\left(\begin{array}{ll}
p^{i-j} & \\
1
\end{array}\right) K_{p} \\
& =\bigcup_{j=0}^{\left\lfloor\frac{\ell}{2}\right\rfloor} Z_{p} K_{p}\left(\begin{array}{ll}
p^{\ell-2 j} & \\
& 1
\end{array}\right) K_{p} .
\end{aligned}
$$

Define $f_{p}: \mathrm{GL}_{2}\left(\mathbf{Q}_{p}\right) \rightarrow \mathbf{C}$ by

$$
f_{p}(g)= \begin{cases}p^{-\ell / 2} & \text { if } g \in M\left(p^{\ell}\right) \\ 0 & \text { otherwise. }\end{cases}
$$

Proposition 7.1 For $f_{p}$ as above, and any newform $f \in S_{k}(N)$, let $\pi_{p}$ be the unramified principal series representation of $\mathrm{GL}_{2}\left(\mathbf{Q}_{p}\right)$ determined by $f$. Then

$$
\widehat{f}_{p}\left(\pi_{p}\right)=X_{\ell}\left(\lambda_{f}(p)\right) \text {. }
$$

Proof Denoting the Satake parameters of $\pi_{p}$ by $\left\{\alpha, \alpha^{-1}\right\}$, we have $\alpha+\alpha^{-1}=\lambda_{f}(p)$. By definition, $\widehat{f}_{p}\left(\pi_{p}\right)$ is the eigenvalue of the operator $\pi_{p}\left(f_{p}\right)$ acting on the unique $K_{p}$-fixed vector of $\pi_{p}$. For the moment, take $f_{p}$ to be the characteristic function of the set $M\left(p^{\ell}\right)$ defined above. It is shown in [KL2, Propositions 4.4-4.5] that, in our current notation, $p^{-\ell / 2} \widehat{f}_{p}\left(\pi_{p}\right)=X_{\ell}\left(\lambda_{f}(p)\right)$. Therefore, upon scaling the characteristic function by $p^{-\ell / 2}$ we get the desired result.

\subsection{The Theorem of Feigon and Whitehouse}

Equation (5.2) of Proposition 5.1 follows immediately from the special case of [FW, Theorem 6.1] given in (7.3) below. Following [FW, \$6.3], we take $F=\mathbf{Q}, \Omega$ trivial, and $N$ prime with $N>D p^{\ell}$ and $\chi(-N)=1$. Then taking $f_{p}$ as in (7.2), [FW, Theorem 6.1] gives

$$
\frac{1}{v(N)} \sum_{f \in \mathcal{F}_{N, k}^{\text {new }}} w_{f} X_{\ell}\left(\lambda_{f}(p)\right)=c_{k} L(1, \chi) \int_{-\infty}^{\infty} X_{\ell} d \eta_{p}^{\chi}
$$

where

$$
c_{k}=\frac{2^{k}}{4 \pi} \frac{\left(\frac{k}{2}-1\right) !^{2}}{(k-2) !}=\frac{k-1}{4 \pi} 2^{k} B\left(\frac{k}{2}, \frac{k}{2}\right)
$$

for the Beta function $B$. (Variants of the exact formula (7.3) can also be found in [MR, FMP, SuT].)

Remarks 7.2 (i) We have adjusted for the fact that we have normalized the completed $L$-functions as in (2.3), whereas the normalization in [FW, p. 407] is twice ours.

(ii) We have also adjusted for the fact that the $L$-value $L(1, \chi)$ is the Dirichlet series (not completed by a Gamma factor), whereas in [FW] the completed $L$-value is used, normalized by $L_{\infty}(1, \chi)=(2 \pi)^{-1}$ as seen in [FW, p. 407]. 
(iii) The lower bound for $N$ of $D p^{\ell}$ comes from the definition of $\mathcal{J}\left(f_{p}\right)$ found in [FW, p. 386]. Since $p+N$, we have $G\left(\mathbf{Q}_{p}\right)=\mathrm{PGL}_{2}\left(\mathbf{Q}_{p}\right)$, and using (7.1) it follows that for our particular test function, $\left|\mathcal{J}\left(f_{p}\right)\right|=p^{\ell}$. This matches [MR, Corollary 1].

\subsection{The Theorem of Ramakrishnan and Rogawski}

As powerful as (7.3) is, it is of interest in some situations to have a formula for the averages in which $N$ is not required to be large in relation to $D$ and $p^{\ell}$. In this range, the error bound given in [FW,MR] is $O\left(N^{-1}\right)$ in the $N$-aspect, so the best bound remains that found in the original paper of Ramakrishnan and Rogawski who obtained (7.3) up to $O\left(N^{-k / 2+\varepsilon}\right)$. By going through their calculations, we will uncover the dependence of the error on both $k$ and $p$. The final result is given in Theorem 7.6.

With the choice of test function (7.2), the spectral side of the relative trace formula in [RR1, Prop. 4.1] becomes

$$
\frac{1}{v(N)} \sum_{f \in \mathcal{F}_{N, k}^{\text {new }}} w_{f} X_{\ell}\left(\lambda_{f}(p)\right)=c_{k} L(1, \chi) \int_{-\infty}^{\infty} X_{\ell} d \eta_{p}^{\chi}+I_{\text {reg }}
$$

for $c_{k}$ as above, where

$$
I_{\text {reg }}=\sum_{x \in \mathbf{Q}-\{0,1\}} I(x)
$$

is the sum of the so-called regular terms, where, for a certain test function $f$ whose local components will be recalled below,

$$
I(x)=\iint_{\mathbf{A}^{*} \times \mathbf{A}^{*}} f\left(\left(\begin{array}{cc}
a b & a x \\
b & 1
\end{array}\right)\right) \chi(a)^{-1} d^{*} a d^{*} b .
$$

Here, we abuse notation and write $\chi$ for the unitary adelic Hecke character determined by the Dirichlet character $\chi$ fixed earlier. The integrals $I(x)$ are computed locally in [RR1, $\$ 2.7$ ] and their sum is bounded in $\$ 3$ of their paper. We shall reexamine these proofs in order to determine the dependence on $p$ and $k$.

The statements of [RR1, Prop. 2.4abcde] each have errors, but this does not affect the validity of the trace formula given in $\$ 5$ of their paper. The following is a corrected version of their proposition.

Proposition 7.3 For $x \in \mathbf{Q}-\{0,1\}$ and $f_{v}$ as in [RR1], define the local integrals

$$
I_{v}(x)=\iint_{\mathbf{Q}_{v}^{*} \times \mathbf{Q}_{v}^{*}} f_{v}\left(\left(\begin{array}{cc}
a b & a x \\
b & 1
\end{array}\right)\right) \chi_{v}(a)^{-1} d^{*} a d^{*} b .
$$

Then the following statements hold.

(a) Let $v=q$ be a finite prime not dividing $p N D$. Then

- $I_{v}(x)=0$ if $v(1-x)>0$;

- if $v(1-x)=0$ and $v(x)=0$, then $I_{v}(x)=1$;

- generally, if $v(1-x) \leq 0$, then

$$
\left|I_{v}(x)\right| \leq \begin{cases}v(x)^{2} & \text { if } v(x) \neq 0, \\ 1 & \text { if } v(x)=0 .\end{cases}
$$

(b) Let $v=q$ be a prime dividing $D$, and write $c=v(D) \geq 1$. Then

$$
\text { - } I_{v}(x)=0 \text { if } v(1-x)>c \text {; }
$$


- if $v(1-x) \leq c$, then

$$
\left|I_{v}(x)\right| \leq 6 q^{c / 2}(2 c+1+|v(x)|) \leq 6 q^{c / 2}(2 c+1)(1+|v(x)|) ;
$$

(c) Let $v=N$. Then $I_{v}(x)=0$ unless $v(x) \geq 1$ (and hence $v(1-x)=0$ ). In this case,

$$
\left|I_{N}(x)\right| \leq v(N)\left|v_{N}(x)\right| .
$$

(d) Let $v=p$, and let $f_{p}$ be the test function defined in (7.2). We suppose $\ell>0$ since the $\ell=0$ case is covered by (i). Then $I_{p}(x)$ vanishes unless $v(1-x) \leq \ell$, in which case

$$
\left|I_{p}(x)\right| \leq 4 p^{-\ell / 2} \ell(\ell+1+|v(x)|) \leq 4 p^{-\ell / 2} \ell(\ell+1)(1+|v(x)|) .
$$

(e) When $v=\infty$,

$$
\left|I_{\infty}(x)\right| \ll \frac{|1-x|^{k / 2}}{|x|}
$$

for an absolute implied constant.

Proof We follow the proof and notation of [RR1]. We begin with part (e), where $f_{\infty}(g)=d_{k} \overline{\left\langle\pi_{k}(g) v, v\right\rangle}$ is the matrix coefficient of the weight $k$ discrete series representation of $\mathrm{PGL}_{2}(\mathbf{R})$ with lowest weight unit vector $v$ and formal degree $d_{k}$. In [RR1, Prop. 2.4e], $I_{\infty}(x)$ is expressed in terms of a certain quantity $I_{\infty}(\varepsilon, \delta, v)$ which is defined as being independent of $x$. This seems to be a typo; as is clear from their proof, $I_{\infty}(x)$ does depend on $x$. But the proof is flawed for other reasons, so we will not try to correct the definition of $I_{\infty}(\varepsilon, \delta, v)$. For $\delta, v \in\{ \pm 1\}$, set

$$
I_{x}^{\prime}(\delta, v)=\int_{0}^{\infty} \int_{0}^{\infty} \frac{a^{k / 2-1} b^{k / 2-1} d a d b}{(a x-v b+\delta i(a b+v))^{k}} .
$$

(This is $I_{\infty}^{\prime}(-v, \delta, v)$ in the notation of [RR1].) Following the proof in [RR1] (we caution that the displayed formula there for $f_{\infty}\left(\left(\begin{array}{cc}a b & a x \\ b & 1\end{array}\right)\right)$ is incorrect), we find, upon observing that $(-1)^{k}=1$ since $k$ must be even, that

$$
I_{\infty}(x)= \begin{cases}d_{k}(2 i)^{k}(1-x)^{k / 2}\left[I_{x}^{\prime}(-1,1)-I_{x}^{\prime}(1,1)\right] & \text { if } 1-x>0, \\ d_{k}(2 i)^{k}(x-1)^{k / 2}\left[I_{x}^{\prime}(-1,-1)-I_{x}^{\prime}(1,-1)\right] & \text { if } 1-x<0 .\end{cases}
$$

As shown in the proof of [RR1, Lemma 7], we have

$$
I_{x}^{\prime}(\delta, v)=B\left(\frac{k}{2}, \frac{k}{2}\right)(\delta i)^{k / 2} J_{x}
$$

where $B(x, y)$ is the Beta function, and

$$
J_{x}=\int_{0}^{\infty} \frac{a^{k / 2-1} d a}{(a x+\delta v i)^{k / 2}(a+\delta v i)^{k / 2}} .
$$

The proof in [RR1] now rotates the line of integration to a purely imaginary ray, overlooking the fact that this ray passes through poles of the integrand in many cases. (Their proof is fixable if one assumes $x>0$, but in fact $I_{\infty}(x)$ need not vanish if $x<0$, despite the assertion to the contrary in [RR1, §3].) The integral $J_{x}$ can presumably be 
computed in terms of special functions even when $x<0$, but since ultimately this integral forms part of an error term, we choose simply to bound it as follows. Observing that $\left|\frac{a}{a \pm i}\right|<1$ for $a>0$,

$$
\begin{aligned}
\left|J_{x}\right| & \leq \int_{0}^{\infty} \frac{d a}{|a x \pm i|^{k / 2}|a \pm i|} \leq \int_{0}^{\infty} \frac{d a}{|a x \pm i|^{k / 2}}=\frac{1}{|x|} \int_{0}^{\infty} \frac{d u}{|u \pm i|^{k / 2}} \\
& =\frac{1}{2} B\left(\frac{1}{2}, \frac{k}{4}-\frac{1}{2}\right)|x|^{-1}
\end{aligned}
$$

by [GR, 8.380.3]. By the above, (7.4), (7.5), and noting that for the standard measure used in [RR1], $d_{k}=\frac{k-1}{4 \pi}$ (cf. [KL1, Prop. 14.4]), we have

$$
\left|I_{\infty}(x)\right| \ll 2^{k} k B\left(\frac{1}{2}, \frac{k-2}{4}\right) B\left(\frac{k}{2}, \frac{k}{2}\right) \frac{|1-x|^{k / 2}}{|x|}
$$

for an absolute implied constant. By Stirling's formula,

$$
B\left(\frac{k}{2}, \frac{k}{2}\right) \sim \frac{2 \sqrt{\pi}}{\sqrt{\frac{k}{2}} 2^{k}} \quad \text { and } B\left(\frac{1}{2}, \frac{k-2}{4}\right) \sim \frac{2 \sqrt{\pi}}{(k-2)^{1 / 2}} .
$$

This gives $\left|I_{\infty}(x)\right| \ll|1-x|^{k / 2}|x|^{-1}$ for an absolute implied constant, which proves assertion (e).

To prove (a) and (d), let $q$ be a prime, fix an integer $r \geq 0$, and let $f_{q}$ be the characteristic function of $Z_{q} K_{q}\left(\begin{array}{c}q^{r} \\ { }_{1}\end{array}\right) K_{q}$. Then $f_{q}\left(\left(\begin{array}{cc}a b & a x \\ b & 1\end{array}\right)\right)$ is nonzero if and only if there exists $\lambda \in \mathbf{Q}_{q}^{*}$ such that $\left(\begin{array}{cc}\lambda a b & \lambda a x \\ \lambda b & \lambda\end{array}\right) \in K_{q}\left(\begin{array}{c}q^{r} \\ { }_{1}\end{array}\right) K_{q}$. By the theory of determinantal divisors ( [Ne, p. 28]), a matrix $g \in \mathrm{GL}_{2}\left(\mathbf{Q}_{q}\right)$ belongs to $K_{q}\left(q^{r}{ }_{1}\right) K_{q}$ if and only if each of the following holds:

- $\operatorname{det} g \in q^{r} \mathbf{Z}_{q}^{*}$,

- each entry of $g$ belongs to $\mathbf{Z}_{q}$,

- some entry of $g$ belongs to $\mathbf{Z}_{q}^{*}$.

(When $r=0$, the third condition is already implied by the first.) So $f_{q}\left(\left(\begin{array}{cc}a b & a x \\ b & 1\end{array}\right)\right) \neq 0$ if and only if there exists $\lambda \in \mathbf{Q}_{q}^{*}$ such that:

(1) $2 v(\lambda)+v(a)+v(b)+v(1-x)=r$,

(2) $v(\lambda)+v(a)+v(b) \geq 0$,

(3) $v(\lambda)+v(a)+v(x) \geq 0$,

(4) $v(\lambda)+v(b) \geq 0$,

(5) $v(\lambda) \geq 0$,

and

(5b) equality occurs in at least one of (2)-(5).

Eliminating $v(\lambda)$, we obtain the following conditions:
(6) $v(a)+v(x)-v(1-x) \geq-r$
(from $(2)+(3)-(1))$,
(7) $v(b)-v(1-x) \geq-r$
(from $(2)+(4)-(1))$,
(8) $v(x)-v(1-x) \geq-r$
(from $(3)+(4)-(1))$,
(9) $v(1-x) \leq r$
(from (1)-(2)-(5)),
(10) $v(a)+v(1-x) \leq r$
(11) $v(b)+v(1-x)-v(x) \leq r$
(from (1)-(4)-(5)),
(12) $v(a)+v(b)+v(1-x) \leq r$
(from (1)-(3)-(5)),
(from (1)-2(5)), 
(13) $v(b) \geq v(a)+v(1-x)-r$

(from 2(4)-(1)).

This leads to the following condensed set of conditions, the last of which is from (5b) and was overlooked in the proof of [RR1, Prop. 2.4]:

(i) $v(1-x) \leq r$,

(ii) $v(x) \geq v(1-x)-r$,

(iii) $v(1-x)-v(x)-r \leq v(a) \leq \min \{r-v(1-x), r-v(1-x)-v(b)\}$,

(iv) $\max \{v(1-x)-r, v(a)+v(1-x)-r\} \leq v(b) \leq v(x)+r-v(1-x)$,

(v) at least one of the following holds:
(va) $v(a)+v(b)+v(1-x)=r$
(if $v(\lambda)=0$, using (1)),
(vb) $v(a)+v(b)-v(1-x)=-r$
(if $(2)=0$, using $2(2)-(1)$ ),
(vc) $v(a)-v(b)+2 v(x)-v(1-x)=-r$
(if $(3)=0$, using $2(3)-(1)$ ),
(vd) $v(b)-v(a)-v(1-x)=-r$
(if (4) $=0$, using $2(4)-(1)$ ).

We may now prove part (a). Suppose $q+p N D$. Then $f_{q}$ is the characteristic function of $K_{q}$ and we can take $r=0$ in the above discussion. The first part of (a) follows from (i). If $r=v(x)=v(1-x)=0$, we see from (iii) and (iv) that $v(a)=$ $v(b)=0$, and since $\chi_{q}$ is unramified and meas $\left(\mathbf{Z}_{q}^{*}\right)=1$, it follows that $I_{v}(x)=1$. Now suppose $v(1-x)<0$. Then $v(x)=v(1-x)$, and (iii) and (iv) become

$$
0 \leq v(a) \leq-v(x), \quad v(x) \leq v(b) \leq 0 .
$$

Using the fact that $\chi_{q}$ is unramified and $\operatorname{meas}\left(\mathbf{Z}_{q}^{*}\right)=1$, we find

$$
\left|I_{v}(x)\right| \leq \sum_{m=0}^{-v(x)} \sum_{n=v(x)}^{0} 1,
$$

and the last assertion of (a) follows in this case. Likewise, if $v(1-x)=0$, then $v(x) \geq 0$, and (iii) and (iv) become

$$
-v(x) \leq v(a) \leq 0, \quad 0 \leq v(b) \leq v(x),
$$

and the assertion holds in this case as well. This proves (a).

Before proving $(\mathrm{d})$, we make some observations about the above conditions for general $r \geq 0$. If $v(1-x) \leq r$, we see from (v) that once $v(a)$ is fixed, there are at most four possibilities for $v(b)$. Setting $m=v(a)$ and $n=v(b)$, we immediately see that

$$
\left|I_{v}(x)\right| \leq \sum_{m=v(1-x)-r-v(x)}^{r-v(1-x)} \sum_{n \in\{4 \text { values }\}} 1=4(2 r-2 v(1-x)+v(x)+1) .
$$

Observing that if $v(1-x)>0($ resp. $v(1-x)=0$, resp. $v(1-x)<0)$, then $v(x)=0$ (resp. $v(x) \geq 0$, resp. $v(x)=v(1-x)$ ); it follows easily that in all cases,

$$
\left|I_{v}(x)\right| \leq 4(2 r+1+|v(x)|) \leq 8(r+1+|v(x)|) .
$$

Now suppose $q=p$ and $f_{p}$ is the test function defined in (7.2). Then by the above, $I_{p}(x)$ vanishes if $v(1-x)>\ell$. When $v(1-x) \leq \ell$, by (7.1), (7.2), and (7.6), we have

$$
\left|I_{p}(x)\right| \leq p^{-\ell / 2} \sum_{j=0}^{\left\lfloor\frac{\ell}{2}\right\rfloor} 8(\ell-2 j+1+|v(x)|) \leq p^{-\ell / 2} \frac{8 \ell}{2}(\ell+1+|v(x)|) .
$$

This proves (d). 
Next, consider $v=N$. Then for

$$
K_{0}(N)_{N}=\left\{\left(\begin{array}{ll}
a & b \\
c & d
\end{array}\right) \in \mathrm{GL}_{2}\left(\mathbf{Z}_{N}\right) \mid c \in N \mathbf{Z}_{N}\right\},
$$

$f_{N}$ is the characteristic function of $Z_{N} K_{0}(N)_{N}$, scaled by $v(N)$. So $f_{N}\left(\left(\begin{array}{cc}a b & a x \\ b & 1\end{array}\right)\right) \neq 0$ if and only if there exists $\lambda \in \mathbf{Q}_{N}^{*}$ such that $\left(\begin{array}{cc}\lambda a b & \lambda a x \\ \lambda b & \lambda\end{array}\right) \in K_{0}(N)_{N}$. The lower right entry must be a unit, which means that in fact we may take $\lambda=1$. Therefore,

$$
\left(\begin{array}{cc}
a b & a x \\
b & 1
\end{array}\right) \in K_{0}(N)_{N}
$$

which means:

$\left(1^{\prime \prime}\right) v(a)+v(b)+v(1-x)=0$,

$\left(2^{\prime \prime}\right) v(a)+v(b)=0$

$\left(3^{\prime \prime}\right) v(a)+v(x) \geq 0$

$\left(4^{\prime \prime}\right) v(b) \geq 1$.

As a result, the integrand vanishes unless:

- $v(1-x)=0$,

- $v(a)=-v(b) \leq-1$,

- $v(x) \geq 1$.

It follows that $I_{N}(x)=0$ unless $v(x) \geq 1$, in which case

$$
\left|I_{N}(x)\right| \leq \sum_{m=-v(x)}^{-1} v(N)
$$

which proves (c).

Lastly, take $v=q$ to be a prime divisor of $D$, and set $c=v(D) \geq 1$. There are some oversights in the definition of the local test function $f_{q}$ at such a place in [RR1, p. 706]: the notation $\chi_{1, v}$ is not defined, $\chi_{v}$ does not define a character of the additive group $X$, and it is asserted that the integral $g\left(\chi_{v}\right)$ defined there, which clearly has absolute value at most 1 , coincides with the classical Gauss sum, which has absolute value $q^{c / 2}$. A detailed treatment of the local test function with the desired spectral properties (and giving the same main term on the geometric side in [RR1]) is given in [JK, (3.11)(3.12)]. For our purpose, it is enough to know that

$$
\operatorname{Supp}\left(f_{q}\right)=\bigcup_{\substack{m \bmod D \mathbf{Z}_{q} \\
q+m}}\left(\begin{array}{cc}
1 & -m / D \\
0 & 1
\end{array}\right) Z_{q} K_{q},
$$

and $f_{q}=\sum_{m} f_{m, q}$, where $f_{m, q}$ is supported on the coset indexed by $m$ and has absolute value $q^{-c / 2}$ there.

To match the notation in [RR1], let $z=m / D(\operatorname{so} v(z)=-c)$ and write $f_{z, v}$ for $f_{m, q}$. Then $f_{z, v}\left(\left(\begin{array}{cc}a b & a x \\ b & 1\end{array}\right)\right) \neq 0$ if and only if there exists $\lambda \in \mathbf{Q}_{q}^{*}$ such that

$$
\left(\begin{array}{ll}
\lambda & \\
& \lambda
\end{array}\right)\left(\begin{array}{cc}
1 & z \\
0 & 1
\end{array}\right)\left(\begin{array}{cc}
a b & a x \\
b & 1
\end{array}\right)=\left(\begin{array}{cc}
\lambda b(a+z) & \lambda(a x+z) \\
\lambda b & \lambda
\end{array}\right) \in K_{q} .
$$

Thus,

(1') $2 v(\lambda)+v(a)+v(b)+v(1-x)=0$

$\left(2^{\prime}\right) v(\lambda)+v(a+z)+v(b) \geq 0$,

(3') $v(\lambda)+v(a x+z) \geq 0$, 
(4') $v(\lambda)+v(b) \geq 0$

$\left(5^{\prime}\right) \quad v(\lambda) \geq 0$,

$\left(5^{\prime} \mathrm{b}\right)$ equality holds in at least one of $\left(2^{\prime}\right)-\left(5^{\prime}\right)$.

As before, we eliminate $v(\lambda)$ to get the following:

$\left(6^{\prime}\right) v(a+z)+v(a x+z)-v(a)-v(1-x) \geq 0$

( $\left.7^{\prime}\right) v(b)+v(a+z)-v(a) \geq v(1-x)$

(8') $v(a x+z)-v(a)-v(1-x) \geq 0$

(9') $v(1-x) \leq v(a+z)-v(a)$

$\left(10^{\prime}\right) v(a)+v(1-x) \leq 0$

$\left(11^{\prime}\right) v(a)+v(b)+v(1-x)-v(a x+z) \leq 0$

(12') $v(a)+v(b)+v(1-x) \leq 0$

(from $\left.\left(2^{\prime}\right)+\left(3^{\prime}\right)-\left(1^{\prime}\right)\right)$,

(from $\left.\left(2^{\prime}\right)+\left(4^{\prime}\right)-\left(1^{\prime}\right)\right)$,

(from $\left.\left(3^{\prime}\right)+\left(4^{\prime}\right)-\left(1^{\prime}\right)\right)$,

(from $\left.\left(1^{\prime}\right)-\left(2^{\prime}\right)-\left(5^{\prime}\right)\right)$,

(from $\left.\left(1^{\prime}\right)-\left(4^{\prime}\right)-\left(5^{\prime}\right)\right)$,

(from $\left.\left(1^{\prime}\right)-\left(3^{\prime}\right)-\left(5^{\prime}\right)\right)$,

$\left(13^{\prime}\right) v(b) \geq v(a)+v(1-x)$

(from $\left.\left(1^{\prime}\right)-2\left(5^{\prime}\right)\right)$,

(from $\left.2\left(4^{\prime}\right)-\left(1^{\prime}\right)\right)$.

(Only $\left(11^{\prime}\right)$ differs from the list in [RR1], whose $\left(11^{\prime}\right)$ seems to be an unmodified paste from (11).) We claim that the above implies the following set of conditions:

(x) $v(1-x) \leq c$,

(y) $v(1-x)-c \leq v(b) \leq v(x)+c-v(1-x)$,

$(\mathrm{z})$ at least one of the following holds:
(zi) $v(a)=-v(1-x)-v(b)$
(if $v(\lambda)=0$, using $\left(1^{\prime}\right)$ ),
(zii) $v(a)+v(1-x)-2 v(a+z)-v(b)=0$
(if $\left(2^{\prime}\right)=0$, using $\left.\left(1^{\prime}\right)-2\left(2^{\prime}\right)\right)$,
(ziii) $v(a)+v(b)+v(1-x)-2 v(a x+z)=0$
(if $\left(3^{\prime}\right)=0$, using $\left(1^{\prime}\right)-2\left(3^{\prime}\right)$ ),
(ziv) $v(a)-v(b)+v(1-x)=0$
(if $\left(4^{\prime}\right)=0$, using $\left(1^{\prime}\right)-2\left(4^{\prime}\right)$ ).

It suffices to prove $(\mathrm{x})$ and $(\mathrm{y})$, since $(\mathrm{z})$ follows from $\left(5^{\prime} b\right)$. To prove $(\mathrm{x})$, if $v(a) \neq v(z)$, then $v(a+z)=\min \{v(a), v(z)\}$, so $v(a+z)-v(a) \leq 0$, which, by $\left(9^{\prime}\right)$, gives $v(1-x) \leq 0<c$. On the other hand, if $v(a)=v(z)=-c$, then by $\left(10^{\prime}\right), v(1-x) \leq c$, as needed.

For $(\mathrm{y})$, note that if $v(a)=v(z)=-c$, then $\left(13^{\prime}\right)$ gives $v(1-x)-c \leq v(b)$ in that case. If $v(a) \neq v(z)$, then as before $v(a+z)-v(a) \leq 0$, and $\left(7^{\prime}\right)$ then gives $v(1-x)-c<v(1-x) \leq v(b)$. This proves the lower bound in (y). For the upper bound, suppose first that $v(a x) \neq v(z)$. Then $v(a x+z)=\min \{v(a x), v(z)\}$, so $v(a x+z) \leq v(a)+v(x)$. (11') then gives $v(b) \leq v(x)-v(1-x)$, which is stronger than the desired upper bound. If $v(a x)=v(z)$, then $\left(11^{\prime}\right)$ is not helpful, because $v(a x+z)=\infty$ is possible. However, in this case, $v(a x)=v(a)+v(x)=-c$, so $\left(12^{\prime}\right)$ gives $v(b) \leq v(x)+c-v(1-x)$, as needed.

Finally, we claim that once $v(b)$ is fixed, there are at most six possible values of $v(a)$ for which $(\mathrm{z})$ is satisfied. It suffices to show that there are at most two possibilities for $v(a)$ if (zii) (resp. (ziii)) is satisfied. Suppose $a$ and $\tilde{a}$ have different valuations and each satisfy (zii). We claim that $v(\tilde{a})=2 v(z)-v(a)$. Write $\tilde{a}=q^{t} u a$ for $u \in \mathbf{Z}_{q}^{*}$ and some integer $t \neq 0$. Then

$$
v(\tilde{a})-2 v(\tilde{a}+z)=v(a)-2 v(a+z),
$$

which gives

$$
v\left(q^{t} u a+z\right)=v(a+z)+\frac{t}{2} .
$$

By Lemma 7.4 below, we get $t=2 v(z)-2 v(a)$, as claimed. For (ziii), by the same argument, we get

$$
v\left(q^{t} u a x+z\right)=v(a x+z)+\frac{t}{2},
$$


so $t$ is again determined by Lemma 7.4: $t=2 v(z)-2 v(a x)$.

By the above discussion, summing over $z$ (i.e., over $\left.m \in\left(\mathbf{Z}_{q} / D \mathbf{Z}_{q}\right)^{*}\right)$, and using $\left|f_{z, v}(g)\right|=q^{-c / 2}$ if nonzero, when $v(1-x) \leq c$ we have

$$
\begin{aligned}
\left|I_{q}(x)\right| & \leq q^{-c / 2} \varphi\left(q^{c}\right) \sum_{n=v(1-x)-c}^{v(x)+c-v(1-x)} \sum_{\{6 \text { values }\}} 1 \leq q^{c / 2} 6(v(x)+2 c-2 v(1-x)+1) \\
& \leq 6 q^{c / 2}(2 c+1+|v(x)|),
\end{aligned}
$$

where the latter inequality is obtained by considering the cases $v(1-x)$ being greater than, equal to, or less than 0 . This proves part (b) of the proposition.

Lemma 7.4 Let $a, z \in \mathbf{Q}_{q}^{*}$ with $a+z \neq 0$, and suppose there exist $u \in \mathbf{Z}_{q}^{*}$ and $t a$ nonzero integer such that

$$
v\left(q^{t} u a+z\right)=v(a+z)+\frac{t}{2},
$$

where $v=v_{q}$. Then $t=2 v(z)-2 v(a)$.

Proof First suppose $v(z)=0$. We need to show that $t=-2 v(a)$. If $v(a)=0$ too, then $v(a+z) \geq 0$, and (7.7) leads to a contradiction if either $t>0$ or $t<0$. Suppose $v(a)>0$, so that $v(a+z)=0$. If $t>-v(a)$, then (7.7) becomes

$$
0=v\left(q^{t} u a+z\right)=\frac{t}{2}
$$

a contradiction. If $t=-v(a)$, then (7.7) becomes

$$
0 \leq v\left(q^{t} u a+z\right)=\frac{t}{2}
$$

which is also a contradiction. If $t<-v(a)$, then (7.7) becomes

$$
t+v(a)=v\left(q^{t} u a+z\right)=\frac{t}{2},
$$

which gives $t=-2 v(a)$. A similar analysis gives the same conclusion if $v(a)<0$.

In the general case, write $z=q^{c} w$ for $w \in \mathbf{Z}_{q}^{*}$. Factoring out $q^{c}$, (7.7) becomes

$$
v\left(q^{t} u \frac{a}{q^{c}}+w\right)=v\left(\frac{a}{q^{c}}+w\right)+\frac{t}{2} .
$$

The special case discussed above then gives $t=-2 v\left(a / q^{c}\right)=2 v(z)-2 v(a)$, as needed.

Proposition 7.5 With local components $f_{v}$ as in Proposition 7.3, the sum of the regular terms is

$$
I_{\text {reg }} \ll \frac{v(N) D^{k}}{N^{k / 2-\varepsilon}} p^{\ell\left(k+\frac{1}{2}\right)},
$$

for any $0<\varepsilon<1$, where the implied constant depends only on $\ell, D$, and $\varepsilon$.

Proof We closely follow [RR1, §3]. Let $M=D p^{\ell}$. Suppose $I(x) \neq 0$. Then by Proposition 7.3, $v_{q}(1-x) \leq v_{q}(M)$ for all primes $q$. This means that $n:=\frac{M}{1-x} \in \mathbf{Z}$. The map $x \mapsto \frac{1}{1-x}$ is a bijection from $\mathbf{Q}-\{0,1\}$ to itself. Therefore, $n$ is not equal to 0 or $M$. Since $N+M$ and $v_{N}(1-x)=0$ by Proposition $7.3 \mathrm{c}$, we have

$$
v_{N}(n-M)=v_{N}\left(M\left(\frac{1}{1-x}-1\right)\right)=v_{N}\left(\frac{x}{1-x}\right)=v_{N}(x) \geq 1,
$$


where the latter inequality is again from Proposition 7.3(c). Thus, $N \mid(n-M)$. Note that $x=\frac{n-M}{n}$. So

$$
I_{\text {reg }}=\sum_{\substack{n \in M+N Z, n \neq 0, M}} I\left(\frac{n-M}{n}\right) .
$$

Since $N+M$, the condition $n \neq 0$ is superfluous. As mentioned earlier, the assertion in [RR1, \$3] that $I_{\infty}(x)=0$ if $x<0$ is incorrect. Now by Proposition 7.3,

$$
I\left(\frac{n-M}{n}\right) \ll p^{-\ell / 2} \sqrt{D} v(N)\left|I_{\infty}\left(\frac{n-M}{n}\right)\right| \prod_{q \mid n(n-M)} B_{q}\left(\frac{n-M}{n}\right),
$$

where

$$
B_{q}\left(\frac{n-M}{n}\right)= \begin{cases}1 & \text { if } v_{q}\left(\frac{n-M}{n}\right)=0 \text { and } q+p D N, \\ v_{q}\left(\frac{n-M}{n}\right)^{2} & \text { if } v_{q}\left(\frac{n-M}{n}\right) \neq 0 \text { and } q+p D N, \\ \left|v_{q}\left(\frac{n-M}{n}\right)\right| & \text { if } q=N, \\ \left(1+\left|v_{q}\left(\frac{n-M}{n}\right)\right|\right) & \text { if } q \mid M\left(=p^{\ell} D\right),\end{cases}
$$

and the implied constant in (7.8) depends only on $\ell$ and $D$.

For the archimedean part, by Proposition 7.3(v) we have

$$
\left|I_{\infty}\left(\frac{n-M}{n}\right)\right| \ll\left|1-\frac{n-M}{n}\right|^{k / 2} \cdot\left|\frac{n-M}{n}\right|^{-1}=\frac{M^{k / 2}}{|n|^{k / 2}\left|1-\frac{M}{n}\right|} .
$$

Observe that for fixed $M,\left|1-\frac{M}{n}\right|$ is as small as possible when $n=M+1$, since $n \neq M$. Hence, $\left|1-\frac{M}{n}\right| \geq \frac{1}{M+1}$. So for an absolute implied constant,

$$
\left|I_{\infty}\left(\frac{n-M}{n}\right)\right| \ll \frac{M^{k / 2+1}}{|n|^{k / 2}} .
$$

To treat the product in (7.8), as shown in the proof of [RR1, Lemma 8], for any $\varepsilon>0$ there exists a constant $C$ depending only on $\varepsilon$ such that

$$
\prod_{q \mid n(n-M)}\left|v_{q}\left(\frac{n-M}{n}\right)\right| \leq C|n|^{\varepsilon}|n-M|^{\varepsilon}
$$

for all $n \neq M$. This in turn is

$$
\ll|n|^{\varepsilon}|n M|^{\varepsilon} \ll|n|^{\varepsilon} M^{\varepsilon}
$$

where not all epsilons are the same, but each may be made arbitrarily small. It follows similarly that

$$
\prod_{q \mid n(n-M)} B_{q}\left(\frac{n-M}{n}\right) \ll|n|^{\varepsilon} M^{\varepsilon}
$$

for any $\varepsilon>0$.

Using (7.9) and (7.10) and recalling that $M=p^{\ell} D$, (7.8) gives

$$
\left|I\left(\frac{n-M}{n}\right)\right| \ll_{D, \ell, \varepsilon} p^{-\frac{\ell}{2}} p^{\ell\left(\frac{k}{2}+1+\varepsilon\right)} D^{k / 2} v(N) \frac{1}{|n|^{k / 2-\varepsilon}} .
$$


So

$$
\begin{aligned}
I_{\text {reg }} & \ll p^{\ell\left(\frac{k+1}{2}+\varepsilon\right)} D^{k / 2} v(N) \sum_{\text {nonzero } m \in \mathbf{Z}} \frac{1}{|M+N m|^{k / 2-\varepsilon}} \\
& =\frac{p^{\ell\left(\frac{k+1}{2}+\varepsilon\right)} D^{k / 2} v(N)}{N^{k / 2-\varepsilon}} \sum_{m \neq 0} \frac{1}{\left|m+\frac{M}{N}\right|^{k / 2-\varepsilon}} .
\end{aligned}
$$

Noting that $\frac{M}{N} \notin \mathbf{Z}$ and $k \geq 4$, the sum is convergent when $\varepsilon<1$. We will show that this sum is $O\left(M^{k / 2-\varepsilon}\right)$.

Generally, for $a>1$ and a noninteger $u>0$ with $u=\lfloor u\rfloor+\{u\}$,

$$
\begin{aligned}
\sum_{m \in \mathbf{Z}} \frac{1}{|m+u|^{a}} & \{u\}^{-a}+(1-\{u\})^{-a}+\sum_{m \geq 1} \frac{1}{|m+\{u\}|^{a}}+\sum_{m \leq-2} \frac{1}{|m+\{u\}|^{a}} \\
& \leq\{u\}^{-a}+(1-\{u\})^{-a}+2 \sum_{m \geq 1} \frac{1}{m^{a}} .
\end{aligned}
$$

We will apply this with $u=\frac{M}{N}$. If $N<M$, then writing $M=q N+r$, we see that $\left\{\frac{M}{N}\right\}=\frac{r}{N} \geq \frac{1}{M-1}$. Likewise, $1-\left\{\frac{M}{N}\right\} \geq \frac{1}{M-1}$, so $\left\{\frac{M}{N}\right\}^{-a}+\left(1-\left\{\frac{M}{N}\right\}\right)^{-a} \leq 2(M-1)^{a}$. If $M<N$, then $\left\{\frac{M}{N}\right\}=\frac{M}{N}$, and the first term in (7.12) comes from $m=0$, which is excluded in (7.11). For the second term, $\left(1-\left\{\frac{M}{N}\right\}\right)^{-a} \leq\left(1-\frac{M}{M+1}\right)^{-a}=(M+1)^{a}$. Taking $a=\frac{k}{2}-\varepsilon$, the third term in (7.12) is $2 \zeta\left(\frac{k}{2}-\varepsilon\right) \leq 2 \zeta(2-\varepsilon)$. It follows that for any prime $N+M$,

$$
\sum_{m \neq 0} \frac{1}{\left|m+\frac{M}{N}\right|^{k / 2-\varepsilon}} \ll M^{k / 2-\varepsilon},
$$

as claimed. With $M=p^{\ell} D$, (7.11) now yields

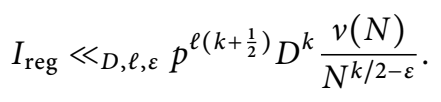

By what we have shown, along with the computation of the main term and measure in [RR1], upon dividing through by $v(N)$ we obtain the following theorem.

Theorem 7.6 Let $k>2$ be an even integer, $\chi=\chi_{-D}$ be as in Theorem 1.3, $N$ a prime not dividing $D$ with $\chi(-N)=-1$, and $p$ a prime not dividing $N D$. Then for all $\ell \geq 0$ and $0<\varepsilon<1$,

$$
\begin{aligned}
& \frac{1}{v(N)} \sum_{f \in \mathcal{F}_{N, k}^{\text {new }}} \frac{\Lambda\left(\frac{1}{2}, f\right) \Lambda\left(\frac{1}{2}, f \times \chi\right)}{\|f\|^{2}} X_{\ell}\left(\lambda_{f}(p)\right) \\
& c_{k} L(1, \chi) \int_{-\infty}^{\infty} X_{\ell} d \eta_{p}^{\chi}+O\left(\frac{p^{\ell\left(k+\frac{1}{2}\right)} D^{k}}{N^{k / 2-\varepsilon}}\right),
\end{aligned}
$$

where $c_{k}=\frac{k-1}{4 \pi} 2^{k} B\left(\frac{k}{2}, \frac{k}{2}\right)$, and the implied constant depends only on $\ell, D$, and $\varepsilon$.

Remarks 7.7 (i) In [RR1], the formula for the formal degree of the weight $k$ discrete series of $\mathrm{PGL}_{2}(\mathbf{R})$ is given as $d_{k}=\frac{k-1}{2}$. This should be corrected to $d_{k}=\frac{k-1}{4 \pi}$, which corresponds to the Haar measure on $\mathrm{SL}_{2}(\mathbf{R})=\mathbf{H} \times \mathrm{SO}(2)$ determined by the measure $\frac{d x d y}{y^{2}}$ on $\mathbf{H}$ and the measure on $\mathrm{SO}(2)$ of total length 1 . 
(ii) For $p$ fixed as above, if $\int X_{\ell} d \eta_{p}^{\chi} \neq 0$, we see from (5.2) that the sum on the left-hand side in Theorem 7.6 is nonzero when $N>p^{\ell} D$. This is stronger than what can be deduced from the above using (7.13) below.

We can now prove (5.1), and so complete the proof of Proposition 5.1. By Theorem 7.6, we have

$$
\frac{1}{v(N)} \sum_{f \in \mathcal{F}} w_{f} X_{\ell}\left(\lambda_{f}(p)\right)=F_{\ell}+E_{\ell}
$$

where $F_{\ell}$ is the main term and $E_{\ell} \ll p^{\ell\left(k+\frac{1}{2}\right)} C_{0}$, where $C_{0}=\frac{D^{k}}{N^{k / 2-\varepsilon}}$. By the proof of Proposition 3.1,

$$
\frac{\sum_{f \in \mathcal{F}} w_{f} X_{\ell}\left(\lambda_{f}(p)\right)}{\sum_{f \in \mathcal{F}} w_{f}}=\int_{-\infty}^{\infty} X_{\ell} d \eta_{p}^{\chi}+O\left(p^{\ell\left(k+\frac{1}{2}\right)} \frac{\frac{C_{0}}{F_{0}}}{1+\frac{E_{0}}{F_{0}}}\right) .
$$

(Note that $F_{0} \neq 0$, since $\int X_{0} d \eta_{p}^{\chi}=1$ as shown in Proposition 6.1.) As noted earlier, $2^{k} B\left(\frac{k}{2}, \frac{k}{2}\right) \sim \frac{2 \sqrt{2 \pi}}{\sqrt{k}}$, so that

$$
c_{k}=\frac{k-1}{4 \pi} 2^{k} B\left(\frac{k}{2}, \frac{k}{2}\right) \sim \sqrt{\frac{k}{2 \pi}} .
$$

Now

and (5.1) follows.

$$
\frac{E_{0}}{F_{0}} \ll \frac{C_{0}}{F_{0}}=\frac{D^{k}}{c_{k} L(1, \chi) N^{k / 2-\varepsilon}} \ll \frac{D^{k}}{k^{1 / 2} N^{k / 2-\varepsilon}},
$$

Acknowledgments We would like to thank the referee for carefully reading the manuscript and offering many suggestions that improved the quality of the results and exposition. We also thank Charles Li, Steven J. Miller, Nigel Pitt, Chip Snyder, and Fan Zhou for numerous helpful conversations.

\section{References}

[BBDDM] O. Barrett, P. Burkhardt, J. DeWitt, R. Dorward, and S. J. Miller, One-level density for holomorphic cusp forms of arbitrary level. Res. Number Theory 3(2017), Art. 25. http://dx.doi.org/10.1007/s40993-017-0091-9

[BBR] V. Blomer, J. Buttcane, and N. Raulf, A Sato-Tate law for GL(3). Comment Math. Helv. 89(2014), no. 4, 895-919. http://dx.doi.org/10.4171/CMH/337

[BLGHT] T. Barnet-Lamb, D. Geraghty, M. Harris, and R. Taylor, A family of Calabi-Yau varieties and potential automorphy II. Publ. Res. Inst. Math. Sci. 47(2011), no. 1, 29-98. http://dx.doi.org/10.2977/PRIMS/31

[Br] R. W. Bruggeman, Fourier coefficients of cusp forms. Invent. Math. 45(1978), no. 1, 1-18. http://dx.doi.org/10.1007/BF01406220

[BrM] R. Bruggeman and R. Miatello, Eigenvalues of Hecke operators on Hilbert modular groups. Asian J. Math. 17(2013), no. 4, 729-757. http://dx.doi.org/10.4310/AJM.2013.v17.n4.a10

[CDF] J. B. Conrey, W. Duke, and D. W. Farmer, The distribution of the eigenvalues of Hecke operators. Acta Arith. 78(1997), no. 4, 405-409. http://dx.doi.org/10.4064/aa-78-4-405-409

[FW] B. Feigon and D. Whitehouse, Averages of central L-values of Hilbert modular forms with an application to subconvexity. Duke Math. J. 149(2009), no. 2, 347-410. http://dx.doi.org/10.1215/00127094-2009-041

[FMP] D. File, K. Martin, and A. Pitale, Test vectors and central L-values for GL(2). Algebra Number Theory 11(2017), no. 2, 253-318. http://dx.doi.org/10.2140/ant.2017.11.253 
[GMR] S. Gun, M. R. Murty, and P. Rath, Summation methods and distribution of eigenvalues of Hecke operators. Funct. Approx. Comment. Math. 39(2008), part 2, 191-204. http://dx.doi.org/10.7169/facm/1229696570

[GR] I. S. Gradshteyn and I. M. Ryzhik, Table of integrals, series, and products. 7th ed., Elsevier/Academic Press, San Diego, 2007.

[Gu] J. Guo, On the positivity of the central critical values of automorphic L-functions for GL(2). Duke Math. J. 83(1996), no. 1, 157-190. http://dx.doi.org/10.1215/S0012-7094-96-08307-6

[ILS] H. Iwaniec, W. Luo, and P. Sarnak, Low lying zeros of families of L-functions. Inst. Hautes Études Sci. Publ. Math. 91(2000), 55-131.

[JK] J. Jackson and A. Knightly, Averages of twisted L-functions. J. Aust. Math. Soc. 99(2015), no. 2, 207-236. http://dx.doi.org/10.1017/S1446788715000142

[KL1] A. Knightly and C. Li, Traces of Hecke operators. Mathematical Surveys and Monographs, 133, American Mathematical Society, Providence, RI, 2006. http://dx.doi.org/10.1090/surv/133

[KL2] Petersson's trace formula and the Hecke eigenvalues of Hilbert modular forms. In: Modular forms on Schiermonnikoog, Cambridge University Press, Cambridge, 2008. http://dx.doi.org/10.1017/CBO9780511543371.011

[KL3] Weighted averages of modular L-values. Trans. Amer. Math. Soc. 362(2010), no. 3, 1423-1443. http://dx.doi.org/10.1090/S0002-9947-09-04923-X

KL4] Kuznetsov's formula and the Hecke eigenvalues of Maass forms. Mem. Amer. Math. Soc. 224(2013), no. 1055. http://dx.doi.org/10.1090/S0065-9266-2012-00673-3

[Ko] E. Kowalski, Families of cusp forms. In: Actes de la Conférence "Théorie des Nombres et Applications", Publ. Math. Besançon Algèbre Théorie Nr., Presses Univ. Franche-Comté, Besançon, 2013, pp. 5-40.

[KS1] N. Katz and P. Sarnak, Random mtrices, Frobenius eigenvalues and monodromy. American Mathematical Society Colloquium Publications, 45, American Mathematical Society, Providence, RI, 1999.

[KS2] Z Zeros of zeta functions and symmetries. Bull. Amer. Math. Soc. 36(1999), 1-26. http://dx.doi.org/10.1090/S0273-0979-99-00766-1

[KST] E. Kowalski, A. Saha, and J. Tsimerman, Local spectral equidistribution for Siegel modular forms and applications. Compos. Math. 148(2012), no. 2, 335-384. http://dx.doi.org/10.1112/S0010437X11007391

[Li1] C. Li, Kuznietsov trace formula and weighted distribution of Hecke eigenvalues. J. Number Theory 104(2004), no. 1, 177-192. http://dx.doi.org/10.1016/S0022-314X(03)00149-5

[Li2] $\longrightarrow$ On the distribution of Satake parameters of $\mathrm{GL}_{2}$ holomorphic cuspidal representations. Israel J. Math. 169(2009), 341-373.

http://dx.doi.org/10.1007/s11856-009-0014-0

[MR] P. Michel and D. Ramakrishnan, Consequences of the Gross-Zagier formulae: stability of average L-values, subconvexity, and non-vanishing mod p. Number theory, analysis and geometry, Springer, New York, 2012, pp. 437-459. http://dx.doi.org/10.1007/978-1-4614-1260-1_20

[Mi] S. J. Miller, An orthogonal test of the L-functions ratios conjecture. Proc. Lond. Math. Soc. (3) 99(2009), no. 2, 484-520. http://dx.doi.org/10.1112/plms/pdp009

[MS] R. Murty and K. Sinha, Effective equidistribution of eigenvalues of Hecke operators. J. Number Theory 129(2009), no. 3, 681-714. http://dx.doi.org/10.1016/j.jnt.2008.10.010

[MT] J. Matz and N. Templier, Sato-Tate equidistribution for families of Hecke-Maass forms on $\operatorname{SL}(n, \mathbf{R}) / \operatorname{SO}(n)$. arxiv:1505.07285

[Na] H. Nagoshi, Distribution of Hecke eigenvalues. Proc. Amer. Math. Soc. 134(2006), no. 11, 3097-3106. http://dx.doi.org/10.1090/S0002-9939-06-08709-0

[Ne] M. Newman, Integral matrices. Pure and Applied Mathematics, 45, Academic Press, New York-London, 1972.

[RR1] D. Ramakrishnan and J. Rogawski, Average values of modular L-series via the relative trace formula. Pure Appl. Math. Q. 1(2005), no. 4, 701-735. http://dx.doi.org/10.4310/PAMQ.2005.v1.n4.a1

[RR2] - Erratum: Average values of modular L-series via the relative trace formula. Pure Appl. Math. Q. 5(2009), no. 4, 1469. http://dx.doi.org/10.4310/PAMQ.2009.v5.n4.a10

[Sa] P. Sarnak, Statistical properties of eigenvalues of the Hecke operators. In: Analytic number theory and Diophantine problems (Stillwater, OK, 1984), Progr. Math., 70, Birkhäuser, Boston, MA, 1987, pp. 321-331. 
[Se] J.-P. Serre, Répartition asymptotique des valeurs propres de l’opérateur de Hecke $T_{p}$. J. Amer. Math. Soc. 10(1997), no. 1, 75-102. http://dx.doi.org/10.1090/S0894-0347-97-00220-8

[ST] S. W. Shin and N. Templier, Sato-Tate theorem for families and low-lying zeros of automorphic L-functions. Invent. Math. 203(2016), no. 1, 1-177. http://dx.doi.org/10.1007/s00222-015-0583-y

[Su] S. Sugiyama, Asymptotic behaviors of means of central values of automorphic L-functions for GL(2). J. Number Theory 156(2015), 195-246. http://dx.doi.org/10.1016/j.jnt.2015.04.003

[SuT] S. Sugiyama and M. Tsuzuki, Relative trace formulas and subconvexity estimates of L-functions for Hilbert modular forms. Acta Arith. 176(2016), no. 1, 1-63.

[T] M. Tsuzuki, Spectral means of central values of automorphic L-functions for GL(2). Mem. Amer. Math. Soc. 235(2015), no. 1110. http://dx.doi.org/10.1090/memo/1110

[W] Y. Wang, The quantitative distribution of Hecke eigenvalues. Bull. Aust. Math. Soc. 90(2014), 28-36. http://dx.doi.org/10.1017/S0004972714000070

[Z] F. Zhou, Weighted Sato-Tate vertical distribution of the Satake parameter of Maass forms on PGL(N). Ramanujan J. 35(2014), no. 3, 405-425.

http://dx.doi.org/10.1007/s11139-013-9535-6

Department of Mathematics and Statistics, University of Maine, Orono, ME 04469-5752, USA

Email: knightly@math.umaine.edu caroline.reno@maine.edu 